ÉGYPTE monde arabe

\section{Égypte/Monde arabe}

$24 \mid 1995$

Anthropologies de l'Égypte 1

\title{
Le « syndicalisme professionnel » en Égypte entre identités socioprofessionnelles et corporatisme
}

\section{Élisabeth Longuenesse}

\section{OpenEdition}

\section{Journals}

Édition électronique

URL : https://journals.openedition.org/ema/647

DOI : 10.4000/ema.647

ISSN : 2090-7273

\section{Éditeur}

CEDEJ - Centre d'études et de documentation économiques juridiques et sociales

\section{Édition imprimée}

Date de publication : 31 décembre 1995

Pagination : 139-188

ISSN : 1110-5097

\section{Référence électronique}

Élisabeth Longuenesse, «Le «syndicalisme professionnel » en Égypte entre identités

socioprofessionnelles et corporatisme », Égypte/Monde arabe [En ligne], 24 | 1995, mis en ligne le 08 juillet 2008, consulté le 07 juillet 2022. URL : http://journals.openedition.org/ema/647 ; DOI : https:// doi.org/10.4000/ema.647

Ce document a été généré automatiquement le 7 juillet 2022.

Tous droits réservés 


\title{
Le « syndicalisme professionnel » en Égypte entre identités socioprofessionnelles et corporatisme
}

\author{
Élisabeth Longuenesse
}

1 Depuis nombre d'années, les «syndicats professionnels " égyptiens font parier d'eux. Ils se sont multipliés à partir des années cinquante, ont eu des relations agitées avec le pouvoir politique pendant la période nassérienne et sont aujourd'hui le théâtre d'intenses batailles entre opposition islamique et pouvoir. Les politologues les ont étudiés à diverses reprises dans la perspective d'une analyse des modes de modernisation de la société, de son contrôle par l'État, de la formation des élites.

2 L'identité des groupes professionnels (c'est-à-dire proclamés tels par la vertu de l'existence du syndicat) qu'ils mobilisent reste cependant incertaine. C'est sur cette question que je voudrais revenir. Les pages qui suivent ne sont qu'une entrée en matière. Dans un premier temps, je passerai rapidement en revue les travaux existants avant de proposer un renouvellement de l'approche, en considérant la question sous un angle sociologique plutôt que politique. Ma réflexion s'inscrit en effet dans le cadre d'une sociologie des groupes professionnels et cherche à mettre à jour ce qui, derrière des désignations communes, fait la spécificité de la situation égyptienne. Puis je reviendrai sur les problèmes de terminologie et de définition avant de faire un bref retour en arrière, qui me permettra de proposer une typologie provisoire de l'ensemble habituellement regroupé sous le qualificatif de «syndicats professionnels » (niqâbât mihaniyya).

3 Je m'étendrai ensuite quelque peu sur la définition juridique du syndicalisme professionnel et sur les aspects formels de leur fonctionnement, en m'efforçant d'en dégager des pistes de réflexion quant à la signification ou aux implications de ces réglementations. Je pars en effet de l'hypothèse que les catégories juridiques ont des effets pratiques en termes de classification et de distinction, autant qu'elles sont 
l'expression de représentations ou de volontés politiques. L'étude empirique doit ensuite montrer comment la réalité sociale s'inscrit dans ces classifications, en tire parti ou au contraire les ignore ou les contourne.

4 Je terminerai en évoquant quelques conflits récents et en m'interrogeant sur la lecture qui peut en être donnée.

Le syndicalisme entre État et société. Pour un renouvellement des paradigmes d'analyse

5 Les travaux qui ont été produits sur les syndicats professionnels en Égypte, essentiellement par des politologues, me paraissent marqués autant par le moment que par le contexte de leur production. J'en proposerai donc dans un premier temps une brève lecture critique, avant de proposer une nouvelle direction de recherche qui tiendra compte à la fois de l'évolution de la réalité et de celle des paradigmes dominants qui tentent d'en rendre compte, précisément en m'intéressant plus à la société qu'à l'État.

Les travaux

6 C'est dans les années soixante-dix que paraissent les premières études portant sur les syndicats professionnels (professionnal syndicates) en tant que tels (Reid, 1974 ; Moore, 1974 ; Springborg, 1978). C'est la grande époque des théories de la modernisation et des nouvelles classes moyennes. La question posée est celle du rôle de ces nouvelles classes dans le processus de modernisation sociale, économique et politique. Le contexte, celui de la libéralisation contrôlée sous Anouar al-Sadate et de l'émergence de nouveaux acteurs. En 1980, Clement H. Moore publie la première édition de son ouvrage sur les ingénieurs, suivi en 1983 par la publication en arabe de celui de Mustafa Kamal alSayyid. Enfin, en 1989, Robert Blanchi renouvelle radicalement l'approche en considérant les syndicats professionnels comme un exemple du phénomène de corporatisation de la société, à côté de la fédération ouvrière, des coopératives agricoles ou mêmes des associations d'hommes d'affaires et des groupes religieux.

7 L'historien Donald Reid comme le politologue Clement H. Moore s'appuient sur le modèle fonctionnaliste classique des professions, définies par un haut niveau de compétence professionnelle, un système de normes et valeurs propres et la priorité de l'évaluation par les pairs; leur analyse se fonde, en dernière instance, sur l'existence d'un modèle occidental (en fait anglo-saxon, ou même américain), et revient à mettre en exergue, sinon expliquer, l'écart par rapport à ce modèle. Le premier, ayant défini des indices de professionnalisation, cherche à repérer leur existence et à les mesurer, ceci dans le cas de cinq professions (droit, médecine, journalisme, génie, enseignement). Mais il conteste l'analyse en termes de classes moyennes - «It would be misleading to suggest that professionals were a cohesive groupe with a shared social outlook »et conclut sur leur échec à fournir l'élite dirigeante du développement économique et social dont le pays avait tant besoin (« they failed to provide constructive leadership for the economic and social development which the country so desperately needed »).

8 Moore, au contraire, se situe dans la lignée des analyses d'Halpem sur la «nouvelle classe moyenne ", définie par ses compétences techniques et sa conscience historique, donc par sa capacité à jouer un rôle dirigeant dans le processus de modernisation; il s'intéresse aux syndicats comme élite professionnelle et s'interroge sur leur aptitude à mettre en œuvre une politique modernisatrice. Son ouvrage, qui porte sur les ingénieurs et non plus sur les syndicats, poursuit et approfondit la réflexion sur la question de la modernisation. Le syndicat est considéré comme outil, lieu et expression 
de la professionnalisation, et comme ayant vocation à être l'organisation d'une élite professionnelle, affirmant une fonction d'expertise et jouant un rôle dans la définition et la mise en œuvre d'une politique. Moore, comme Reid, conclut sur l'échec des syndicats à réaliser une telle ambition modernisatrice.

9 Springborg déplace l'angle de l'analyse. Il ne parle plus de modernisation mais de développement, et considère les syndicats professionnels comme lieu d'observation du «développement politique " (ici synonyme de «modernisation» politique), comme «baromètre du changement à l'intérieur de l'élite »: c'est donc aussi en politologue, s'intéressant aux processus d'émergence des élites politiques et à leur dynamique interne, qu'il les étudie. Ce faisant, son cadre conceptuel est radicalement différent de celui des auteurs précédents : son objet n'étant pas le syndicat en tant que tel (ni même la profession), mais les élites, il peut prendre en considération les différentes conceptions en jeu, les batailles autour de l'existence de ces syndicats, les efforts des acteurs politiques pour les instrumentaliser, mais aussi - donc - pour les modeler en vue de servir un certain projet social et politique (en particulier, les enjeux du débat concernant l'alternative intégration des professionnels dans le syndicalisme ouvrier versus organisation d'une élite professionnelle), alors que ses prédécesseurs étaient prisonniers d'un modèle, d'une conception monolithique de la profession comme élite, que le pouvoir s'efforçait de dénaturer pour la contrôler. Il considère le syndicat non comme objet de son étude, mais comme champ et enjeu d'une lutte politique.

Bianchi se situe dans la même lignée fonctionnaliste, imposée par la réalité du fonctionnement des organisations professionnelles. La nouveauté de son apport est l'introduction du paradigme corporatiste. Toutefois, comme ses prédécesseurs, il continue à centrer son analyse sur les rapports entre organisations syndicales et pouvoir, plus qu'entre celles-ci et la société, et fait largement l'impasse sur les contradictions internes à ces organisations, les tiraillements concernant leur nature, etc. Il part d'une interrogation sur la validité des paradigmes mis en œuvre à partir du modèle latino-américain dans l'analyse du "développement dépendant ", c'est-à-dire de ce que certains auteurs ont défini comme un « syndrome périphérique » dans lequel développement dépendant, autoritarisme et corporatisme seraient liés. Le corporatisme étant considéré comme un instrument du contrôle social dans un système autoritaire, et les organisations syndicales devenant des outils d'encadrement de la société, Bianchi montre le caractère contradictoire du phénomène corporatiste en Égypte, à la fois instrument de contrôle et lieu de cristallisation d'une opposition. L'originalité de son analyse est non seulement de centrer son propos sur le phénomène corporatiste, mais aussi d'abandonner l'américano-centrisme de ses prédécesseurs. Il n'est plus - il est moins ?- prisonnier du modèle occidental et a renoncé à la problématique de la modernisation ou du développement.

11 Il en reste cependant au niveau de la forme des organisations et ne s'interroge pas sur leur relation avec la société elle-même (les groupes et catégories sociales qu'elles sont censées représenter). Tout à sa préoccupation de montrer le processus de " corporatisation » des organisations syndicales (synonyme d'une prise de contrôle par le pouvoir), il gomme les contradictions et les hésitations, et voit un processus linéaire et continu là où Springborg notait les divergences et les luttes entre conceptions différentes. Inversement, il suit ensuite l'évolution des organisations professionnelles, qui se sont transformées en tremplin pour une opposition au régime, sans s'interroger sur les éventuelles contradictions internes à ces organisations. Ce qu'il peut faire en en restant 
à une analyse événementielle de leur action. Du coup, il réduit l'analyse en traitant de la même manière des organisations fort différentes à l'origine: le phénomène de corporatisation ne me semble pas en effet avoir le même sens pour les syndicats ouvriers et pour des organisations dont la logique, le principe d'origine, sont corporatistes.

Mustafa Kamal al-Sayyid, dès 1983, avait introduit le concept de "groupe d'intérêt ", qui sera repris par Amâni Qandîl dans le Rapport Stratégique d'al-Ahrâm à partir de 1986. Sous ce même chapeau, il regroupe toutes les organisations syndicales et professionnelles, ainsi que les associations de tout acabit, des hommes d'affaires aux associations religieuses. Son souci est de montrer comment, derrière la façade du parti au pouvoir, une multiplicité d'intérêts continue, bon an mal an, à s'exprimer. À la différence des auteurs étrangers, il ne cherche pas à tester la validité et l'universalité d'une grille d'analyse élaborée ailleurs. II instrumentalise un concept emprunté à la politologie américaine pour mesurer et analyser les modes d'expression autonomes de la société face à l'État. Ce faisant, il annonce les débats qui domineront quelques années plus tard, avec la montée du courant islamique, sur l'affirmation de la société civile face à l'État. Il conclut que la tendance à la politisation des syndicats n'est que le résultat des limites imposées à l'expression démocratique par le système politique.

Aujourd'hui, alors que les syndicats les plus prestigieux sont passés les uns après les autres aux mains de directions islamistes, les politologues égyptiens situent tous leurs études sur le syndicalisme professionnel, implicitement ou explicitement, dans le cadre de la réflexion sur l'affirmation nouvelle de la société civile face à l'État. Nadia AbuGhâzi (1992), dans un article sur le syndicat des artistes, considère leur lutte comme "symbolique de la nouvelle conscience démocratique de certains secteurs de la société égyptienne » et s'y intéresse de ce point de vue ${ }^{1}$. Amâni Qandîl, qui a tenu la chronique sur les "groupes d'intérêts " dans le Rapport stratégique d'al-Ahrâm de 1986 à 1991 et publié un certain nombre d'articles sur la question, s'attache à l'analyse du phénomène islamiste dans les syndicats, à leurs méthodes et aux raisons de leur succès. Si elle ne manque pas d'établir une relation entre ce succès et les difficultés croissantes des " classes moyennes ", son intérêt reste limité aux formes de l'action politique. Mustafa Kamal al-Sayyid, dans un récent numéro de Maghreb-Machrek, pousse plus loin l'analyse de l'évolution de la situation des ingénieurs et de leurs problèmes et de la manière dont la direction du syndicat les prend en charge. Mais la problématique de ces auteurs reste centrée sur l'étude des modes de mobilisation des groupes professionnels dans la bataille pour la démocratie.

Pour un renouvellement de l'approche

Les travaux cités, privilégiant un questionnement politique, ont en commun de ne pas mettre en question l'identité des groupes concernés. Or, à y regarder de plus près, on est frappé par l'hétérogénéité de l'ensemble de ces groupes désignés, sous le qualificatif de "professionnels » (mihaniyyîn), et par la variabilité des frontières entre les uns et les autres selon les périodes (voir plus loin).

Mon projet vise donc à déplacer le regard vers la société en me demandant sur quel type d'identité sociale s'appuient ces organisations et dans quelle mesure elles contribuent ou non à les cristalliser. Mon point de départ est celui d'une sociologie des groupes professionnels telle que de récents travaux français et étrangers tentent de la renouveler. 

la suite de Talcott Parsons est marquée par le paradigme fonctionnaliste ; elle propose un modèle de la profession définie par une compétence spécialisée acquise lors d'une formation longue, combinée à une vocation de service public et un esprit de corps. La médecine en offre un archétype à l'aune duquel sont évaluées les autres professions (cf. Dubar, 1991, chapitre 6). La tradition française, partant de la sociologie du travail, a une conception plus large de la profession, considérée essentiellement comme catégorie statistique. Un courant récent propose un renouvellement de la réflexion sous la forme d'une sociologie de la professionnalisation et de la construction des identités professionnelles qui croise l'héritage précédent avec une réflexion sur la formation et la qualification et s'appuie sur une démarche résolument comparatiste ${ }^{2}$. C'est de ces derniers travaux que je m'inspire, non pas tant pour tenter d'appliquer un modèle peu pertinent dans le contexte égyptien que pour les questionnements qu'ils me suggèrent.

En effet, parce que les conditions historiques de formation du marché du travail, de structuration de la société, d'émergence des élites, de constitution du politique sont, en Égypte, totalement différentes de ce qu'elles sont dans les pays développés, on ne peut, sans risquer le contre-sens, s'aventurer à transposer tels quels les paradigmes d'une sociologie des groupes professionnels, même renouvelée, au cas égyptien. Inversement, le discours sur les syndicats professionnels tel qu'il s'est développé depuis une vingtaine d'années dans ce pays est nécessairement marqué par les conditions spécifiques dans lesquelles il est produit. La différence entre le cas égyptien (ou celui de tout autre pays dépendant, ou anciennement colonisé) et le cas français (ou américain, ou de tout autre pays européen industrialisé) est qu'il se dédouble en un discours d'origine exogène et un discours d'origine endogène, le premier ayant plus d'influence sur le second que l'inverse. Dans les deux cas toutefois, le fait de parler des syndicats, plus que des groupes professionnels eux-mêmes, renvoie à la prééminence des enjeux politiques dans la réflexion des chercheurs.

18 Les travaux des chercheurs du Cedej, en privilégiant une approche génétique (historique) par laquelle ils cherchent à dévoiler les mécanismes d'émergence et de construction des pratiques et des institutions «modernes", offrent l'exemple d'une réflexion s'efforçant, loin des schémas a priori, de rendre compte de ce qui fonde l'originalité de la réalité égyptienne et de la rendre intelligible avant de s'aventurer dans des généralisations hâtives ${ }^{3}$.

C'est entre ces deux pôles -celui du questionnement d'une sociologie spécialisée et celui d'une analyse concrète respectueuse de la réalité étudiée - que j'essaie de développer ma réflexion en gardant en permanence à l'esprit la nécessité d'interroger les conditions spécifiques de construction de l'objet étudié et de mise en œuvre, implicite ou explicite, des paradigmes d'analyse. Partant de là, mon postulat de départ est que le système des "professions " à l'égyptienne est le produit d'un double mouvement d'importation et d'acclimatation d'un modèle étranger et de production endogène de pratiques et d'institutions répondant à la dynamique propre de la société.

20 Les hypothèses que je propose privilégient la dimension symbolique, mais aussi l'analyse des conflits et de leurs enjeux. Pierre Tripier, à l'issue d'une exploration rigoureuse des paradigmes fondateurs de ce qu'il définit comme la matrice disciplinaire de la sociologie du travail, conclut: "Si le monde est fait à partir d'interactions, le passé est toujours présent, avec ses légendes, ses mythes, son histoire reconstruite, ses normes et ses prescriptions, mais la créativité individuelle, de nouvelles rencontres 
bouleversent à tout moment l'ordre des choses. Toute théorie générale de la société devient alors inutile... Seule la recherche de terrain, le stock de connaissances amassées dans des protocoles rigoureux, peut permettre des généralisations circonspectes d'une réalité aussi susceptible de changements... » Et plus loin : «Ainsi, à défaut de dire la vérité sur le monde, peut-on dire la vérité sur la conception que les hommes s'en font. » (Tripier, 1991, p. 178) En m'inspirant de sa méthode, je me propose d'orienter ma recherche à partir de la double interrogation suivante :

-Quelle est la signification de cette forme spécifique de « syndicalisme », quels sont ses effets en termes d'identité sociale, à quels enjeux est-elle confrontée, dans quel sens évolue-t-elle? Pourquoi, mais aussi comment et, surtout, dans quelle mesure cette forme d'organisation contribue-t-elle ou non à cristalliser des solidarités ?

- Comment se traduit (quels sont les effets de) l'hétérogénéité croissante de cette population de professionnels et de diplômés?

Cette interrogation est guidée par deux hypothèses complémentaires :

- Si la corporatisation du mouvement syndical est sans aucun doute très largement le produit d'une volonté politique (thèse de Bianchi), l'élargissement du syndicalisme professionnel à des catégories de plus en plus larges de diplômés, sur la base du diplôme plutôt que sur celle d'une pratique professionnelle clairement identifiée, serait l'expression d'une volonté de distinction, d'une revendication de reconnaissance de la part de ces diplômés, les plaçant au-dessus de la masse des travailleurs non qualifiés. Ce faisant, je ne fais que reprendre à mon compte des remarques déjà faites par certains auteurs comme Moore qui, en 1974, évoquait déjà la « dynamique irrésistible qui poussait étudiants et familles à revendiquer l'égalité des chances et l'équivalence entre instituts et université ", ou Springborg, qui suggérait que les syndicats professionnels permettaient à des groupes de statut inférieur à l'intérieur de la profession - essentiellement les salariés du secteur public - de se faire entendre (1978, p. 278-279).

- Les syndicats professionnels sont aujourd'hui le lieu d'un conflit multiforme - d'intérêts, de culture, etc. - entre anciennes élites professionnelles et nouveaux diplômés. Ce conflit se manifeste à l'intérieur des syndicats les plus anciens (ingénieurs, médecins, juristes, diplômés de commerce) et entre ceux-ci et les nouveaux syndicats créés dans les années soixante-dix (entre les ingénieurs et les syndicats de professions techniques...). Les changements successifs de législation, ceux qui modifient le fonctionnement d'un syndicat, comme ceux qui créent de nouveaux syndicats, en sont une expression et marquent les étapes de cette lutte.

Au début de cette étude, il était nécessaire de faire le point sur ce qui est désigné sous la catégorie de syndicalisme professionnel en Égypte et sur les conditions historiques de son émergence. C'est l'objet des pages qui suivent.

Question de terminologie

Le paysage syndical égyptien se répartit entre deux grands ensembles d'organisations :

- une fédération syndicale ouvrière (al-ittihâd al-'amm li niqâbât al-'ummâl) regroupant un ensemble de syndicats de branche, dont les adhérents sont employés dans un type donné d'entreprise, quelle que soit la nature de leur qualification et de leur travail ;

- un nombre croissant d'organisations dites « syndicats professionnels » (niqâbât mihaniyya), regroupant en principe chacun un secteur de la population active défini par la qualification et le métier, quel que soit le secteur ou le type d'emploi ; ils sont créés par décision législative. 
Cedeuxième type d'organisation, malgré la même désignation en arabe (le terme niqâba est utilisé dans les deux cas, spécifié par l'adjectif 'ummâliyya, ouvrier, ou mihaniyya, professionnel), est régi par un ensemble de textes spécifiques définissant des missions assez différentes de celles d'un syndicat ouvrier. Nombre d'auteurs écrivant en français préfèrent les désigner comme «ordres professionnels». Une telle option peut se justifier au moins pour deux raisons :

- l'antériorité des organisations professionnelles d'avocats, de médecins, d'ingénieurs et de pharmaciens, qui eurent d'abord les fonctions d'un ordre tel que la tradition des professions libérales les connaît en France, et qui servirent de modèle aux suivants ;

- plus fondamentalement (ceci découlant de cela), quelques-unes des caractéristiques de ces organisations les rapprochent de l'ordre : leur création par voie législative, l'obligation d'adhésion, leur rôle dans la définition de règles pratiques et morales codifiant la pratique professionnelle, la fonction disciplinaire ${ }^{4}$.

Mais l'«ordre professionnel» est de conception typiquement française; hérité de l'ancien régime ${ }^{5}$, il est caractéristique des professions libérales. Il a pour fonction première le contrôle des conditions d'exercice, donc d'accès à la profession, la définition d'un code de déontologie et le maintien de la discipline; l'adhésion y est obligatoire. Au contraire, la tradition anglo-saxonne respecte scrupuleusement le principe de la liberté d'adhésion, y compris pour des organisations qui jouent par ailleurs un rôle essentiel dans le contrôle des conditions d'exercice d'une profession et dans la définition des diplômes et des titres y donnant accès. L'Angleterre comme les États-Unis en effet ne connaissent pas l'ordre mais seulement l'association professionnelle, à laquelle l'adhésion est libre ${ }^{6}$. En revanche, aux États-Unis, les "professions" sont reconnues comme telles par une législation spécifique qui réglemente leurs relations avec la société7.

est vrai que les ordres professionnels à la française ont une autre référence, moins glorieuse, celle du corporatisme fasciste ${ }^{8}$. Mais ce modèle concernait l'ensemble des « corps » socio-professionnels, y compris les ouvriers, dont les syndicats ne sont pas pour autant considérés comme des ordres 9 .

Dans le cas des organisations professionnelles égyptiennes telles qu'elles se sont développées depuis les années cinquante, se sont en outre adjointes un certain nombre de fonctions de nature économique et sociale, qui relèvent de l'entraide ou de la défense de la profession et ne sont pas traditionnellement associées à l'idée d'ordre. Pour toutes ces raisons, les désigner par le terme d'« ordre » me paraît peu pertinent et susceptible de prêter à confusion.

La terminologie anglaise en matière de syndicalisme a des connotations encore différentes, héritées d'une tout autre histoire. Le terme qui s'est imposé pour désigner les syndicats ouvriers étant celui d'union, les auteurs anglo-saxons, pour rendre la spécificité du syndicat professionnel égyptien, ont choisi d'exhumer le vieux terme syndicate - " groupe de gens qui coopèrent dans un but requérant des fonds importants et qui ont mis des ressources en commun $»^{10}-$, ce qui le . rapproche de la notion de corps ou de corporation.

29 Ces difficultés terminologiques produisent des flottements récurrents: la presse francophone ou anglophone parlera parfois indifféremment, dans un même article, d'ordre ou de syndicat pour l'une, de union ou de syndicate pour l'autre ou, selon les 
journalistes, choisira l'un ou l'autre terme. Pour le lecteur averti, c'est sans doute sans grande importance. Pour le profane, la confusion est en revanche totale ${ }^{11}$.

Mon parti pris est donc, dans la mesure du possible, de m'en tenir à la manière dont les intéressés se désignent en conservant les ambiguïtés produites par l'usage arabe d'un terme unique, niqâba; je le traduirai donc dans tous les cas par syndicat, quitte à examiner dans un second temps les équivoques qui en découlent du fait du parallèle implicite avec d'autres situations, en particulier occidentales. Quelques exemples illustreront le sens de ce choix : lorsque, en 1961, lors de la réunion préparatoire au Congrès national des forces populaires, Gamal Abdel Nasser s'attaque aux syndicats professionnels, un de ses arguments est l'impossibilité, ou l'absurdité, de regrouper dans une même organisation employeurs et employés : ce faisant, sa référence est bien le syndicat ouvrier ${ }^{12}$.

De même, quand le journal al-Wafd du 15/02/95 propose un historique du mouvement syndical, il commence par les guildes ${ }^{13}$, continue avec les syndicats ouvriers puis débouche sur la situation d'aujourd'hui, qu'il caractérise par l'existence de "trois sortes de groupes d'intérêt (syndicats), professionnels, ouvriers, patronaux»: si l'analyse distingue trois sortes de syndicats, tous ont une filiation unique, le syndicat ouvrier, lui-même héritier des corporations...

Enfin, dans un autre registre, lorsque le journal al-Ahrâr du 16/02/95 annonce en gros titre "Grève générale des syndicats", il parle évidemment - dans l'Égypte d'aujourd'hui, personne ne le comprend autrement - des syndicats professionnels : cependant, je ne peux m'empêcher de lire, dans une telle annonce, une référence symbolique aux moments forts du mouvement syndical ouvrier. Un plus bel exemple encore est donné par le journal al-Sha'b du 30/09/94 dans un petit article sur le " Rôle des syndicats dans le mouvement national égyptien", signé d'un universitaire, 'Abd Allah Shâmi, dont nous donnons de larges extraits en annexe.

Il me reste à dire un mol du concept de "groupe d'intérêt " emprunté à la science politique américaine et introduit par Mustafa Kamal al-Sayyid dans un ouvrage paru en 1983 (voir supra). Le Rapport stratégique d'al-Ahrâm l'adopte en 1986, au lieu de " groupes de pression " utilisé dans le même sens l'année précédente: sont compris dans cet ensemble les syndicats et les associations de toutes sortes, professionnelles, culturelles, caritatives ou autres, c'est-à-dire toute forme de regroupement institutionnalisé visant à prendre en charge et à défendre les intérêts d'un groupe quel qu'il soit. Je préfère pour ma part ne pas l'utiliser, car en rassemblant sous un même vocable des organisations extrêmement diverses, représentant des intérêts trop différents et parfois même opposés, il me parait relever d'un choix fonctionnaliste qui aboutit à brouiller les enjeux véritables de leur activité et contribuer, de ce fait, à obscurcir l'analyse plutôt qu'à l'éclairer.

Un historique du syndicalisme professionnel

34 Le paysage syndical tel qu'il se présente à nous aujourd'hui est composé de strates superposées, constituées à des moments historiques différents, de sorte que les enjeux de la création des différents syndicats étaient chaque fois différents.

- La question de l'héritage des corporations ottomanes est posée par certains auteurs. Donald Reid pense que s'il n'y a pas d'emprunt explicite, il ne fait guère de doute que le modèle corporatif traditionnel a influencé les nouvelles organisations professionnelles (Reid, 1974, p. 37). Quant à Robert Blanchi, s'appuyant sur Gabriel Baer, il suggère que les similitudes ne sont pas le simple fait du hasard (Baer, 1989, p. 60 et s.). Baer avait en effet montré que les 
corporations égyptiennes (à la différence des syriennes) avaient acquis, tout au long du XIX ${ }^{\mathrm{e}}$ siècle, de nouvelles fonctions qui en faisaient les intermédiaires de l'État pour le contrôle du marché du travail, de l'application des réglementations, le prélèvement des taxes, etc. (Baer, 1969, p. 151). Avec le développement de l'appareil administratif, elles disparaîtront progressivement (ibid. p. 159).

- Les premières organisations professionnelles naissent dans le contexte de la lutte nationale pour défendre les droits des nationaux face aux praticiens ou aux experts étrangers. Le syndicat des avocats, le plus ancien, est créé en 1912 sur le modèle du barreau des tribunaux mixtes qui date de $1876{ }^{14}$ (Reid, 1974, p. 40). En 1920, médecins et ingénieurs font une première tentative, avortée, de se donner une association professionnelle nationale. Il faudra attendre 1940 pour obtenir la légalisation des premiers, 1946 pour celle des seconds. Bianchi souligne le caractère contradictoire des relations de ces organisations avec le pouvoir à l'époque libérale : si l'instrumentalisation des organisations professionnelles et syndicales par les parts politiques et particulièrement le Wafd, dans l'entre-deux-guerres, est sans doute un frein à l'autorisation de nouvelles associations (Blanchi, 1989, p. 66), inversement, les créations des années quarante (journalistes, médecins, pharmaciens, vétérinaires) sous le gouvernement de 'Ali Maher, parallèlement à celle du ministère des Affaires sociales, correspondent clairement, selon le même auteur, à une volonté de contrôle social (ibid. p. 72).

- Le pouvoir nassérien poursuit la « corporatisation » de la société en supprimant toute forme de pluralisme et en attribuant de nouvelles fonctions aux organisations professionnelles et syndicales. « Nasser ne crée pas le corporatisme, il en hérite, mais l'étend à de nouveaux secteurs » (ibid., p. 77). L'« innovation est l'élaboration d'une vision organique de la société » (ibid., p. 79). Il unifie le syndicalisme ouvrier en remplaçant la multitude de syndicats faiblement reliés en une fédération regroupant un petit nombre de syndicats fortement centralisés.

Mais tandis que Blanchi insiste sur le projet nassérien de corporatisation de la société, Springborg montre que celui-ci ne s'est pas imposé sans conflit ni hésitations : en effet, les relations entre les syndicats professionnels et le pouvoir ont été cahotiques et conflictuels tout au long de la période, et l'on a assisté à l'affrontement de logiques contradictoires, qui ont différemment prévalu selon les moments.

La première décennie (1952-61) est marquée par l'ambivalence du pouvoir à l'égard des syndicats, par les mesures administratives, la manipulation des élections, la promotion de leaders proches du régime, sans que soit remise en cause la nature des syndicats ni même réellement leur autonomie. Au début des années soixante, le conflit se radicalise; médecins, avocats et journalistes se battent pour défendre l'indépendance syndicale; les médecins luttent contre la socialisation de la médecine ; Nasser dénonce le caractère bourgeois des syndicats. La mise en place de l'Union socialiste arabe en 1964 marque un tournant; Nasser pose alors dans ces termes, rapportés par Springborg, la question de l'utilité des syndicats : «L'existence des syndicats est-elle nécessaire? L'Union socialiste arabe (USA) est la principale organisation et les membres des syndicats sont membres de l'USA. La question est alors: devons-nous supprimer l'USA, ou les syndicats? » Mais la gauche de l'USA réagit en défendant au contraire l'idée du rôle nécessaire des organisations professionnelles dans la mobilisation pour le socialisme (Springborg, 1978, p. 286-287). Cette hésitation entre une logique de classe (suppression des organisations bourgeoises) ou une logique corporatiste (les transformer en instrument de mobilisation) se résout en faveur de la 
seconde option. Mais celle-ci se double du même coup d'un effort de "démocratisation" par l'élargissement à de nouvelles catégories moins qualifiées (donc moins prestigieuses), en abaissant le niveau du diplôme exigé à l'entrée du syndicat: en 1966, une réforme du syndicat des agronomes l'élargit aux techniciens diplômés des instituts agricoles ; le syndicat des avocats s'ouvre en 1968 aux conseillers juridiques salariés du secteur public. En revanche, les ingénieurs et les médecins résistent avec succès à l'élargissement de leur syndicat, pour les premiers aux techniciens, pour les seconds aux infirmières et autres professions paramédicales (Moore, 1974, p. 27).

37 - Les années Sadate se caractérisent par une politique d'ouverture économique qui s'accompagne d'une brève tentative de libéralisation politique; mais l'agitation qui s'ensuit provoque un raidissement du pouvoir, une volonté renforcée de contrôle politique et un recours accru aux systèmes d'allégeance personnelle (Waterbury, 1983, chapitre 15). De nouveaux syndicats sont créés, de nouvelles lois sont promulguées. Apparaissent ainsi successivement le syndicat des "commerciaux " (diplômés des facultés et instituts de commerce) en 1972, le syndicat des professions sociales en 1973, le syndicat des techniciens (professions techniques appliquées) en 1974, puis le syndicat des diplômés des instituts d'arts appliqués en 1976 et le syndicat des professions soignantes en $1976^{15}$. On peut faire l'hypothèse que les créations syndicales des années soixante-dix sont à la fois dans le droit fil des évolutions en cours depuis les années soixante, et l'indice de nouveaux développements liés aux réorientations politiques amorcées par Sadale, tant dans le domaine économique que dans le domaine politique et institutionnel.

38 Un responsable du syndicat des ingénieurs analyse la période de Sadate, en ces termes (il parle en 1995): "On peut appeler l'époque de Sadate époque de l'ouverture économique (infitâh) ou époque de l'utilitarisme (intifâ'), au sens où les dirigeants politiques ont eu soin de récompenser les syndicats qui faisaient allégeance au pouvoir. » (Muhammad 'Ali Bishr, Congrès des syndicats professionnels, 08/04/95)

- La présidence de Hosni Moubarak voit se poursuivre ce même mouvement contradictoire :

une libéralisation économique qui s'accompagne de mesures nouvelles en faveur d'un pluralisme de l'expression politique, mais aussi d'une répression accrue des forces contestataires et de toute tentative de jonction entre contestation politique et mouvement social. De même, les syndicats se trouvent tiraillés entre crispation corporatiste et politisation extrême. Les effectifs connaissent une croissance inégalée, et la différenciation sociale interne à la population des professionnels et diplômés est croissante ${ }^{16}$.

39 En 1986, pour la première fois, le syndicat des médecins se donne une direction islamiste. Les ingénieurs suivent leur exemple un an plus tard, puis les scientifiques, les pharmaciens et, plus récemment, les avocats. Le courant islamiste voit son influence grandir dans les syndicats professionnels, en particulier les plus anciens. Cependant, jusqu'à présent, par consensus entre toutes les forces en présence, la fonction de président reste entre les mains de personnalités du pouvoir, ou proches de lui (voir A. Qandil, 1994, p. 283).

De nouveaux syndicats sont créés dans les années quatre-vingts et quatre-vingt-dix, dans une logique que l'on est tenté de décrire comme purement corporatiste.

Pour une typologie des syndicats professionnels

41 Le tableau publié en annexe donne une liste des syndicats professionnels existants, de leur date de création, de leurs effectifs et des textes législatifs régissant leur 
fonctionnement. Cet ensemble se caractérise par la grande variété des catégories représentées: de la profession libérale - ou de tradition libérale - clairement identifiée (avocats, médecins) à l'association de diplômés (des facultés de commerce, d'écoles et instituts techniques industriels), en passant par les métiers artistiques ou diverses professions salariées (enseignants, infirmières...).

Catégories en fonction du type de métier ou d'emploiLes professions libérales en voie de salarisation

Un certain nombre de ces syndicats - les plus anciens - regroupent des professions à l'origine libérales, bien que comptant un nombre de plus en plus grand de salariés: c'est le cas des avocats, des professions de santé (médecins, dentistes, vétérinaires, pharmaciens) ainsi que des comptables, dont l'organisation est créée un peu plus tard, en 1955. Professions indépendantes, ayant affaire à une clientèle personnelle et non à l'État ni à des sociétés ou organismes publics ou privés, ce sont elles qui fournissent le modèle de la "profession » et de l'organisation professionnelle; cette dernière a pour première fonction de définir et réglementer les conditions de la pratique, plus encore que celles de l'accès au marché. À l'origine existait également une mission de défense et de protection des nationaux face à la concurrence des étrangers (Chiffoleau, 1992; Botiveau, 1994). Elles connaissent aujourd'hui un double processus de salarisation et de paupérisation relative qui produit des clivages très forts en leur sein et affaiblit l'esprit de corps, la solidarité professionnelle et, du même coup, l'identité professionnelle.

Les professions d'experts au service de l'État

Ingénieurs et agronomes ont une position différente des précédents: sauf pour une minorité (les architectes, inclus dans l'ensemble "ingénieur», ainsi que quelques ingénieurs-conseil), il ne s'agit pas de professions libérales mais plutôt de professions d'expertise, constituant des corps au service de l'État; cependant, comme les précédentes, elles ont une identité professionnelle historiquement forte et prestigieuse et, de ce point de vue, s'en rapprochent. Leurs organisations, créées dans les années quarante sur le modèle des précédentes, avaient de même une double finalité de défense des nationaux en même temps que de promotion de la profession et de développement d'un esprit de corps (Moore, 1980).

Eux aussi connaissent un processus de déclassement par élargissement. À côté d'une petite élite de hauts fonctionnaires, mais aussi de cadres dirigeants du secteur privé, se développe une masse de cadres salariés de l'administration, des services publics, etc. Dans les années soixante, ils ont subi une forte pression de la part des métiers techniques ayant un moindre niveau de qualification et qui demandaient leur intégration ; les ingénieurs résistèrent victorieusement à ces pressions, les techniciens devant se contenter d'un statut d'observateur pendant quelques années avant d'obtenir, sous la présidence d'Anouar al-Sadate, la création de leur propre syndicat (Moore, 1974, p. 30 et 1980, p. 59); au contraire, les agronomes durent accepter l'association de tous les diplômés d'écoles agricoles (Springborg, 1978, p. 288 ; Moore, 1980 , p. 58), le titre d'ingénieur agronome restant protégé et accessible aux techniciens au bout de dix ans de pratique.

Les professions du spectacle et les métiers de la culture

Les professions artistiques telles que comédiens, musiciens, métiers du cinéma... doivent gérer les conditions d'embauche : la réglementation du marché du travail et de l'accès à la profession est une fonction essentielle de leurs syndicats. 

même époque (respectivement en 1973 et en 1976). Les premières avaient eu dans les années quarante, un syndicat qui avait participé aux luttes ouvrières de l'époque. Ce syndicat avait disparu avec la centralisation syndicale des années cinquante, sous Nasser, tandis que les infirmiers et infirmières se retrouvaient au sein du syndicat de la santé, avec l'ensemble des personnels dépendant du ministère de la Santé, qualifiés et non qualifiés. Dans les années soixante, il avait été question de fondre l'ensemble des professions de santé, de l'infirmière au médecin (Moore, 1974), dans un seul syndicat, selon le principe qui poussait au regroupement des techniciens agricoles et des agronomes, des diplômés d'écoles et d'instituts industriels et des ingénieurs. On a vu plus haut que seuls les premiers avaient obtenu gain de cause. La création d'un syndicat professionnel relève donc d'une tout autre logique, ayant entre autres choses une forte valeur symbolique, de reconnaissance d'une distinction par rapport à la masse des salariés non diplômés, et de rapprochement à l'égard des professions les plus prestigieuses. Inversement, un tel processus de distinction peut aussi avoir un effet de séparation, c'est-à-dire de division des différentes catégories de salariés, à moins qu'il n'en soit la conséquence. Pour les professions sociales, indissociablement liées aux projets réformistes (Roussillon, 1994), la création d'un syndicat a sans doute une signification plus politique de mobilisation et d'encadrement

Les diplômés à faible définition professionnelle

52

part au précédent, sauf que le contour en est flou et la définition des métiers encore davantage: les scientifiques, les 
commerciaux, les professions techniques. Leurs syndicats sont créés à des époques très diverses et regroupent des catégories ayant une place dans la société, un statut, un prestige très différents les uns des autres. Leur caractère commun est d'avoir pour critère d'identification un diplôme donnant accès à des métiers et des emplois trop divers pour que puissent véritablement se développer des solidarités professionnelles au sens strict.

Les «scientifiques"( al-mihan al-'ilmîyya) regroupent tous les diplômés - niveau licence, ou bakâlûrius, en quatre ans et plus - des facultés de sciences, quelque soit leur emploi, sauf les enseignants du secondaire: on trouve donc parmi eux des chercheurs dans divers organismes publics et ministères et des enseignants du supérieur. Ils revendiquent un prestige égal à celui des ingénieurs et se plaignent de ne pas bénéficier des mêmes avantages et de la même reconnaissance.

Les «commerciaux» (tugâriyyîn) sont tous les diplômés des facultés de commerce, y compris économie et science politique. Leur syndicat a été constitué en phagocytant le petit syndicat des experts-comptables, qui était quant à lui un véritable syndicat professionnel constitué sur le modèle d'un ordre de profession libérale. Aujourd'hui, les diplômés de commerce se comptent par centaines de milliers et occupent diverses catégories d'emplois de cadres de gestion et d'administration (outre les postes de comptables); ils se trouvent souvent en concurrence avec diverses sortes de diplômés, en particulier de langue, du fait de la faible définition des postes et de la logique de fonctionnement du marché de l'emploi, où les relations comptent souvent davantage que la compétence ${ }^{17}$. D'où un mélange de frustration massive et une absence de solidarité professionnelle encore plus grande que dans d'autres catégories.

Les professions techniques (al-mihan al-fanniyya al-tatbîqîyya) regroupent les diplômés des écoles et instituts industriels, quel que soit leur emploi, public ou privé, en entreprise, dans l'administration ou dans le secteur artisanal. La création de leur syndicat aboutit à «distinguer » les ouvriers qualifiés (en fait, diplômés) des autres ouvriers, et elle est considérée par les militants ouvriers comme une opération de division de la classe ouvrière.

Les nouveaux métiers

56 Dans les années quatre-vingts, de nouveaux syndicats sont créés dans des domaines très divers : des lecteurs du Coran (1983) aux sportifs (1987) en passant par les guides touristiques (1983). En 1993 est créé le syndicat des physiothérapeutes. Plus récemment, un syndicat des «ingénieurs technologues» aurait obtenu sa reconnaissance (al-Wafd, 29/08/94 et 26/09/94). D'autres groupes réclament leur syndicat: les pédagogues (al-Sha'b, 26/05/95), les archéologues (al-Akhbâr, 26/08/94), etc. Il y a là sans doute conjonction de plusieurs facteurs favorisant une logique de morcellement corporatiste à la base, n'ayant plus grand chose à voir avec le corporatisme de l'époque nassérienne : effet, d'un côté, de l'aggravation des conditions d'emploi, durcissant la concurrence, mais aussi, de l'autre, d'une politique de libéralisation au moins formelle facilitant la multiplication des organisations sociales, culturelles ou syndicales.

Les associations à vocation syndicale

Ces «syndicats professionnels », constitués sur la base d'un métier, d'une qualification ou d'un diplôme, n'ont pas une véritable vocation à prendre en charge les intérêts de groupes de salariés définis par une position commune sur le marché de l'emploi. Une seule exception: les enseignants, tous employés et rémunérés par le ministère de 
l'Éducation. Mais il est frappant que ni le syndicat des ingénieurs, ni celui des commerciaux, ni celui des professions techniques - pour prendre trois exemples parmi d'autres - ne sont en mesure de fournir des statistiques précises sur l'emploi de leurs adhérents, sur la proportion de ceux qui travaillent dans le secteur public ou dans le secteur privé, dans telle ou telle branche ou secteur d'activité.

Inversement, si dans la plupart des entreprises ou organismes, publics ou privés, c'est le syndicat ouvrier qui est censé se préoccuper de questions de salaires ou de conditions de travail ${ }^{18}$, certaines institutions manquent de véritable structure syndicale. C'est par exemple le cas des enseignants du supérieur qui, pour pallier ce manque, ont créé en 1974, au plus fort des luttes étudiantes et de l'influence de la gauche dans les universités, des associations, les « clubs des corps enseignants " (nâdî hay'at al-tadrîs); ces " clubs " sont régis par la loi de 1964 sur les associations, ce qui en principe devrait leur interdire toute action syndicale. L'adhésion y est facultative. À la même époque, de façon plus discrète, les chercheurs du Centre national de la recherche se donnaient une structure équivalente, le «club des corps de la recherche " (nâdî hay'at al-bahth al-'ilmî).

Les relations avec le syndicalisme ouvrier

$\mathrm{Si}$, sauf exception, on ne peut en principe adhérer à deux syndicats professionnels ${ }^{19}$, il est au contraire normal et bienvenu d'être membre à la fois d'un syndicat professionnel et d'un syndicat ouvrier. Dans les entreprises publiques, l'adhésion au syndicat ouvrier est automatique et la cotisation est prélevée à la source, avant versement du salaire, sauf demande expresse du salarié concerné. Ainsi les ingénieurs, techniciens, juristes, comptables ou même médecins travaillant dans une entreprise adhèrent-ils généralement au syndicat dont relève leur entreprise. C'est que les deux types de syndicalisme ne relèvent pas, en principe, de la même logique et ne sont pas censés avoir la même fonction. Les relations entre employés et employeurs ne relèvent que du comité syndical d'entreprise (structure de base du syndicat ouvrier), et le syndicat professionnel n'a pas à intervenir à ce niveau. Le seul interlocuteur du second est l'État, par exemple sur des questions de statut, donc concernant essentiellement la fonction publique. Dans les faits, pas plus le syndicat ouvrier que les syndicats professionnels ne jouent le rôle de porte-parole des luttes revendicatives, comme on le constate périodiquement lorsque éclatent des mouvements de grève ou de protestation: ils se font tous en dehors de lui, voire contre lui ${ }^{20}$. En revanche, comme les syndicats professionnels, les comités syndicaux d'entreprise sont des prestataires de services, d'aides sociales et médicales diverses, d'organisation de loisirs, etc. Mais outre cela, ils ont des représentants au conseil d'administration de l'entreprise et les élus syndicaux jouent un rôle de médiateur entre l'employé et le patron. Ils ont donc une position stratégique et, à la différence des dirigeants des syndicats professionnels, sont connus des salariés. Selon des témoignages divers, c'est ce qui explique que la participation de ces derniers aux élections syndicales soit forte et que les professionnels ne restent pas à l'écart.

60 Pour autant, ceux-ci ne sont pas tout à fait considérés comme des salariés semblables aux autres : cela se traduit par une sous-représentation dans les conseils syndicaux. En effet, l'art. 35 du code du travail promulgué en 1976 (deux ans après la création du syndicat des professions techniques appliquées) limite à $20 \%$ la représentation des "professionnels» dans les conseils syndicaux ${ }^{21}$. Lors des élections syndicales, cela signifie que si, par exemple, pour 20 postes à pourvoir, plus de 4 «professionnels » se 
trouvent bénéficier d'un nombre de voix suffisant pour être élus, le cinquième et les suivants seront déclassés au profit d'ouvriers se trouvant en $21^{\mathrm{e}}$ position d'éligibilité ou plus: c'est souvent ce qui se passe. Avec la création du syndicat des professions techniques (diplômés d'écoles et instituts industriels), c'est parfois la moitié des salariés d'une entreprise industrielle (par exemple la Société du fer et de l'acier de Helwan) qui entrent dans la catégorie de "professionnels » et se trouvent donc sousreprésentés dans les instances dirigeantes du syndicat.

61 Le 15 avril dernier, le Conseil constitutionnel s'est avisé qu'une telle restriction était anticonstitutionnelle, car contraire à l'égalité des citoyens et à la liberté syndicale ${ }^{22}$. Ce jugement faisait suite à une plainte déposée, après les élections de 1991, par une salariée appartenant à la catégorie des « professionnels » et qui avait obtenu le nombre de voix nécessaire pour être élue, mais avait été déclassée en faveur d'un « ouvrier ». Il concluait à la nullité des élections syndicales depuis 1976 et à l'obligation de procéder dans les plus brefs délais à de nouvelles élections.

Le syndicalisme professionnel : missions et fonctionnement

Définies par la loi, les missions du syndicat n'ont cependant pas toutes la même réalité et leur importance a pu varier selon les époques. Dans ce qui suit, nous allons passer en revue les principaux aspects du fonctionnement dès syndicats en mettant en valeur leurs caractéristiques communes et en relevant les spécificités de certaines professions. Pour illustrer notre propos, nous nous étendrons plus particulièrement sur deux exemples, a priori assez différents, celui des ingénieurs et celui des enseignants, sans nous interdire d'en évoquer d'autres. Il ne s'agit donc que d'un premier dépouillement indicatif; un travail d'analyse comparative systématique des textes pourrait être faite apporterait, me semble-t-il, un éclairage intéressant à la compréhension des enjeux sous-jacents à la définition de la nature de ces organisations et de leurs relations avec l'État.

63 Chaque syndicat est régi par une législation spécifique, construite à peu près sur le même modèle, qui en définit les missions (représentation et promotion de la profession ou du groupe, protection d'un titre, mission d'expertise) ainsi que les conditions d'adhésion, l'organisation et le mode de fonctionnement, le régime financier et la gestion des caisses de retraite, les règles disciplinaires; la présentation. le volume, le développement des différents chapitres varient selon les cas, peut-être en fonction de la date de promulgation, mais également selon la nature du groupe concerné, professionnels ou diplômés.

Objectifs et fonctions

64 "The raison d'être of syndicates is to protect the professional interests of their members, including salaries, pensions, conditions of work and regulation of entry into the profession. » (Springborg, 1978, p. 278) Cette affirmation est en fait un raccourci plus ou moins approximatif selon les périodes et les syndicats. II est tout à fait intéressant et instructif de revenir à ce qui apparaît dans les textes juridiques comme la mission officielle du syndicat, qui varie non seulement selon la nature du syndicat mais, plus encore, selon le moment où il est fondé, et de la comparer avec la pratique réelle du syndicat et avec la vision qu'en ont les adhérents.

Le syndicat des ingénieurs (loi $n^{\circ} 66$ de 1974, amendée par la loi $n^{\circ} 7$ de 1983) voit ses objectifs ainsi définis :

Art. 1: II est fondé un syndicat, dit syndicat des ingénieurs, bénéficiant de la personnalité morale, considéré comme l'instance de représentation des ingénieurs 
de nationalité égyptienne et comme organe consultatif auprès de l'État dans le domaine de sa spécialité (...).

Art. 2 : Le syndicat travaille à la réalisation des objectifs suivants :

- élever le niveau scientifique et professionnel des ingénieurs, protéger l'honneur de la profession, définir et appliquer les règles appropriées à l'organisation de la pratique de la profession et à l'accomplissement, par les membres du syndicat, de leurs devoirs au service du pays, et contrôler leur application ;

- mobiliser les membres du syndicat (...) au service de la société (...) ;

- développer l'esprit de fraternité et d'entraide parmi les membres, œuvrer à l'amélioration de leur niveau professionnel, social et matériel, assurer leur vie et prendre soin de leur famille sur les plans social, économique, sanitaire, culturel ;

- contribuera l'étude des plans de développement économique et aux projets industriels et technologiques ;

- contribuer à la définition des programmes de formation technique et d'ingénieurs (...) ;

- travailler à la diffusion et au développement des recherches et études (etc.).

Pour les enseignants (loi ${ }^{\circ} 79$ de 1969), on note quelques différences :

Art.1 : II est fondé un syndicat pour les métiers de l'enseignement, bénéficiant de la personnalité morale, qui englobe tous ceux qui travaillent dans le métier (mihna) de l'éducation et de l'enseignement, ou qui ont travaillé antérieurement dans ces métiers, et dont l'activité est placée sous le signe de la politique générale de l'Union socialiste arabe $(. . .)^{23}$

Art. 2 : Le syndicat agit pour la réalisation des objectifs suivants ;

- se mettre au service de la société pour réaliser ses objectifs nationaux, et plus particulièrement :

a) mobiliser ses membres au service de la société ;

b) travailler à diffuser la culture et l'enseignement ;

c) contribuer aux plans de développement et aux projets éducatifs (...)

- travailler à élever le niveau de la profession, et en particulier :

a) défendre l'honneur de la profession, élever le niveau des enseignants et leur compétence;

b) contribuer au développement de l'enseignement ;

c) encourager la publication et le développement scientifique (...) ;

d) nouer des liens avec les organisations d'enseignants.

- offrir des services aux membres, et en particulier :

a) des services économiques, sociaux, culturels et de loisirs ;

b) une aide (financière) en cas de besoin ;

c) des soins de santé ;

d) la gestion de pensions de retraite, d'invalidité et de décès...

67 On note qu'il est question de promotion de la profession, de contribution à l'organisation de la profession (pour certaines professions comme les ingénieurs), à la définition des programmes de formation, et aussi d'entraide, de services, mais pas à proprement parier de " protection des intérêts professionnels, salaires ou conditions de travail " (selon les termes de Springborg). Si, dans la pratique, au cours de la dernière période, les syndicats professionnels n'ont pas été sans s'en préoccuper, il me paraît intéressant de noter que ce type d'action n'est pas inscrit dans leurs missions telles que définies initialement par la loi.

Sur un autre plan, il existe aussi des différences notables d'un syndicat à l'autre, dont les deux cas ici choisis donnent un exemple : assez remarquablement, on relèvera que le syndicat des ingénieurs bénéficie de la qualité d'instance de représentation des ingénieurs et d'organe consultatif auprès de l'État, ce dont le syndicat des enseignants, en revanche, n'est pas gratifié. Comme si le second n'avait ni autonomie ni personnalité 
propre, et en était réduit à des fonctions d'encadrement et de mobilisation pour la mise en œuvre d'un projet qu'il n'est pas censé contribuer à définir. L'énumération des missions du syndicat et l'ordre dans lequel elles sont présentées va dans le même sens : à travers la vocation à "définir et appliquer les règles (...) de la pratique ", les ingénieurs se voient reconnaître un contrôle de la profession sur elle-même ${ }^{24}$ dont les enseignants sont privés. Inversement, si, dans les deux cas, le syndicat a une mission de mobilisation au service de la société, cette mission apparaît prioritaire pour les enseignants, et l'insistance y est plus marquée dans leur cas.

À côté des objectifs ainsi définis, les syndicats ont deux fonctions importantes, celles que l'on attribue habituellement à un «ordre » : la protection du titre et le contrôle de l'accès à la profession, d'une part, et, d'autre part, la fonction disciplinaire.

Titre et expertise

L'existence d'un titre protégé est mentionné pour six professions : les avocats - art. 2, loi $\mathrm{n}^{\circ} 17$ de 1983 -, les médecins - art 11 . loi n ${ }^{\circ} 415$ de $1954{ }^{25}$-, les ingénieurs - art 98, loi $\mathrm{n}^{\circ} 66$ de 1974 -, les professions agricoles (l'usage du titre d'ingénieur agricole est défini par l'art 4 et protégé par l'art 90 de la loi $n^{\circ} 31$ de 1966), les professions techniques appliquées (qui bénéficient d'un titre de "spécialiste » - akhissầ - défini et protégé par les art 103 et 104 de la loi $n^{\circ} 67$ de 1974), enfin les diplômés d'arts appliqués (art 97, loi n 84 de 1976). Cette question du titre peut être associée à celle de la protection d'un marché de l'emploi, mais elle a aussi une fonction symbolique ${ }^{26}$. Sa signification n'est évidemment pas du tout la même pour un médecin et pour un... technicien. Dans un cas, il s'agit de freiner le déclin provoqué par l'accroissement numérique et la transformation des conditions de travail et d'emploi; dans l'autre, à l'inverse, il s'agit de chercher la reconnaissance d'une distinction par rapport au commun des salariés ou des fonctionnaires peu qualifiés. Dans trois cas, la législation attribue au syndicat une mission d'expertise auprès de l'État; il s'agit des ingénieurs, techniciens et diplômés d'arts appliqués, auxquels un article de loi donne expressément une telle mission (cf. plus haut, l'art. 1 de la loi sur le syndicat des ingénieurs). Une telle qualité n'est mentionnée ni pour les médecins ni pour les diplômés de commerce, dont on aurait pu penser qu'ils peuvent être consultés ou sollicités pour la définition des politiques de santé, ou pour les finances ou l'économie, par exemple. Cependant, les syndicats professionnels ont, à diverses périodes, été sollicités ès-qualité : ainsi, en 1979, est formé un «conseil des naqî̉ » que le président consulte pour les décisions importantes (al-Ahrâm, 15/08/79). Cette qualité d'expertise peut être considérée de deux points de vue complémentaires : du point de vue de l'État, comme l'expression de l'effort pour se donner les moyens de contrôler ses ressources économiques et humaines ${ }^{27}$; du point de vue de la société, comme la légitimation du pouvoir d'une élite.

Conditions d'appartenance et obligation d'adhésion

71 L'adhésion est en principe obligatoire pour exercer (mais pour exercer quoi ?) - ou pour prétendre à être recruté sur un poste correspondant à la qualification et au titre possédés ; cependant cette obligation n'est pas toujours explicitée de la même façon. Inversement, la condition nécessaire et suffisante pour adhérer est généralement de posséder le diplôme donnant accès à l'exercice de la profession considérée. Le diplômé de médecine ou de génie civil doit adhérer pour pouvoir exercer, s'il ne réussit pas à vivre de l'exercice de sa profession et se trouve réduit à exercer une autre activité, il peut rester membre. La plupart des syndicats distinguent en effet deux catégories 
d'adhérents : les membres actifs et les non actifs. Dans d'autres cas, l'adhésion implique l'exercice, comme chez les enseignants (voir plus bas), ou dans des professions où le métier ne correspond pas à un diplôme précis, comme pour les journalistes ou les professions artistiques; il y a alors une période intermédiaire, de mise à l'épreuve, où le candidat ne peut travailler qu'avec l'autorisation du syndicat, mais ne devient membre à part entière qu'après un certain temps d'activité. Pour les avocats, il y a une période de stage, principe que certains souhaiteraient voir étendre à d'autres professions ${ }^{28}$.

L'adhésion signifie inscription sur un registre donnant le droit d'exercer. Les syndicats tiennent en général au moins deux registres: l'un pour les membres "actifs », l'autre pour les « non-actifs ». Parfois, les « actifs » se répartissent entre plusieurs registres en fonction du niveau ou du type de qualification: ainsi les professions agricoles ont-elles deux registres, un pour les ingénieurs agronomes, un autre pour les techniciens agricoles $^{29}$.

Dans la pratique, l'obligation d'adhésion n'a donc pas le même sens et ne s'applique pas de la même façon, selon la réalité des conditions d'emploi des différentes catégories considérées, selon que la profession a les moyens de contrôler l'accès au marché du travail (professions indépendantes) ou non (professions salariées); selon que l'emploi dépend de l'État ou du secteur privé; selon qu'il s'agit de professions clairement définies ou de diplômés aux métiers mal définis. Trois exemples illustreront ces différences ;

- Pour les enseignants, l'obligation est relativement facile à appliquer : « L'adhésion est obligatoire pour tous ceux qui travaillent dans le métier (mihna) de l'éducation (tarbiyya) et de l'enseignement (ta'lîm), à l'exception des membres du corps enseignant des universités azhari, des instituts supérieurs, des facultés et des écoles militaires, pour lesquels elle est facultative » (art. 4, loi 79 de 1969), et la cotisation est prélevée sur le salaire par l'organisme pour lequel travaille l'adhérent (art. 13). C'est bien l'activité qui est première et qui détermine l'adhésion. L'introduction de l'obligation d'adhésion au syndicat, comme pour le syndicat ouvrier, avait dans ce cas le sens d'une corporatisation d'une profession qui ne l'était pas auparavant, à la différence des vieilles professions.

- Pour les ingénieurs au contraire, l'obligation d'adhésion est beaucoup plus ancienne et a une signification différente, liée à la protection du titre et au contrôle de l'emploi. L'article 7 de la loi 66 de 1974 énonce : « II est interdit aux ministères, aux organismes, entités, régies, institutions publiques, aux sociétés et aux individus, d'employer pour une fonction d'ingénieur, ou de passer contrat pour un travail d'ingénieur, avec une personne ou avec un bureau de conseil en ingénierie qui ne serait pas inscrit sur les registres du syndicat (...) », tandis que l'article 3 impose l'obligation " pour les universités, facultés et instituts supérieurs (...) de transmettre au syndicat la liste des diplômés, le niveau de leur diplôme et le lieu de résidence dans les 60 jours suivant la proclamation des résultats des examens ", et que l'article 98 prévoit une peine pouvant aller jusqu'à 6 mois de prison pour quiconque utiliserait indûment le titre d'ingénieur ou réaliserait des travaux d'ingénieur sans être inscrit au syndicat. Dans les faits, il existe sans doute une certaine proportion d'ingénieurs diplômés non inscrits (cf. al-Sayyid, 1994 ; Hanali, 1995). Il n'en reste pas moins que c'est bien le diplôme qui conditionne l'adhésion et qu'il s'agit au départ d'un moyen de contrôle de la profession sur elle-même, même si ce contrôle est négocie avec la puissance publique.

- Le syndicat des commerciaux offre un troisième cas de figure : non seulement la législation n'impose aucune obligation de ce genre, mais le règlement intérieur prévoit même la possibilité d'accueillir en son sein les diplômés d'autres facultés qui exerceraient des 
travaux entrant dans le cadre des spécialisations admises par le syndicat ${ }^{30}$. Quant à la masse des diplômés de commerce, ils souffrent surtout de l'absence de véritables définitions de postes correspondant à leurs compétences et, de notoriété publique, ils seraient des milliers à ne pas être inscrits au syndicat ${ }^{31}$.

Déontologie et fonction disciplinaire membres » (chap. 5 de la loi $n^{\circ} 66$ de 1974 sur le syndicat des ingénieurs, chap. 3 de la $2^{\mathrm{e}}$ partie de la loi $n^{\circ} 79$ de 1969 sur le syndicat des enseignants, etc.). Ce chapitre s'ouvre sur le serment que doit prononcer le nouvel adhérent au moment de son adhésion devant le conseil du syndicat. Les ingénieurs prononcent le serment suivant : " Je jure devant Dieu d'accomplir les tâches de mon métier dans la loyauté et l'honneur, de protéger le secret du métier et son honneur, d'en respecter les lois et les traditions " (art. 52 de la loi $n^{\circ} 66$ de 1974); si l'évocation des «secrets du métier » rappelle les règlements corporatistes, elle ne correspond guère aux réalités de la pratique des ingénieurs, ni même à celles des architectes, aujourd'hui de plus en plus souvent salariés, donc n'ayant pas la propriété de leur travail. Celui des enseignants diffère précisément sur ce point, remplacé par le respect du " pacte de l'enseignant arabe »: «Je jure devant Dieu d'accomplir mon travail dans la loyauté et l'honneur, de respecter les principes du pacte de l'enseignant arabe, de respecter les lois et les us du métier » (art. 10 de la loi $n^{\circ} 79$ de 1969). Il faudra donc se reporter au texte du pacte en question pour en évaluer la signification.

Les autres devoirs concernent le paiement de la cotisation, l'information faite au syndicat de tout changement de situation, le respect de certaines règles dans les relations avec les autres membres : exigences fort disparates, relevant de la loyauté à l'égard du syndicat pour les uns, à l'égard des collègues pour les autres. et les devoirs, elle relève donc de la corporation et non d'une instance qui serait définie en fonction du lieu de travail ou du type d'emploi. On est une fois de plus tenté d'y voir soit la survivance d'une logique corporatiste pré-moderne, soit une volonté de corporatisation, de cristallisation d'un " esprit de corps ", chaque groupe assumant une fonction dans la société, par laquelle il contribue à l'intérêt général. C'est cet effort de définir des règles déontologiques et d'organiser un contrôle disciplinaire du groupe sur lui-même, manifestation de la volonté du législateur de promouvoir un corporatisme professionnel, qui amène à qualifier ces organisations d'ordres plutôt que de syndicats.

Il reste à vérifier si ces instances disciplinaires fonctionnent réellement et dans quelles circonstances elles sont utilisées. On peut supposer qu'elles ne le sont qu'assez rarement, sachant qu'il existe par ailleurs des instances disciplinaires dans les institutions publiques, où sont employés le plus grand nombre de ces « professionnels ». L'enquête reste à faire sur ce point, et il serait intéressant de vérifier quelle instance est sollicitée, dans quel cas, et s'il peut y avoir conflit entre elles.

Aide sociale et caisses de retraite

Dans les faits, la mission la plus visible au quotidien pour l'adhérent est celle qui concerne les différentes formes d'aide sociale, et d'abord - et surtout - la gestion d'une caisse de retraite. Il est frappant qu'un chapitre important de chaque législation syndicale détaille le fonctionnement de la caisse de retraite et son mode de financement ${ }^{32}$. Si sa gestion est distinguée dans l'ensemble du budget syndical, elle n'en relève pas moins du syndicat lui-même et non d'un organisme autonome et la plus 
grosse part des ressources du syndicat y sont consacrées (voir plus loin). Le niveau des pensions versées est variable d'un syndicat à l'autre et dépend du niveau de ressource de chaque syndicat. Ces pensions viennent compléter celles qui sont versées par l'État ou par l'entreprise pour les salariés. Dans le contexte de baisse du pouvoir d'achat des salaires et des retraites, il s'agit, même si le montant n'en est pas très élevé, d'un avantage non négligeable qui fait des "professionnels» salariés des privilégiés par rapport aux autres salariés.

Structure et organisation

Le «syndicat général» (niqâba 'âmma) regroupe des sections (firq) syndicales, ou syndicats de province: en général un par province, sauf exception ${ }^{33}$. Par ailleurs, le syndicat est subdivisé, au niveau national, en branches (shu'ab) de spécialité : les ingénieurs ont sept «branches» (génie civil, architecture, mécanique, électricité, chimie, textile, mines), de même que les commerciaux (comptabilité, gestion, économie, statistique commerciale, science politique, assurance, métiers connexes), mais les enseignants n'en ont pas.

Les sections locales, de même que les branches, élisent chacune un conseil, lequel envoie des représentants au conseil du syndicat général. Celui-ci est composé, d'une part, de délégués des sections et branches, d'autre part, de membres élus au niveau national. Toutes les instances dirigeantes sont élues par des assemblées générales, locales ou nationales. Les conditions d'éligibilité sont plus ou moins rigoureuses d'un syndicat à l'autre : 15 ans d'ancienneté pour tous les membres du conseil chez les ingénieurs, de même que pour les présidents des sections locales ou des branches (art. 9 de la loi 66 de 1974), pour les présidents de sections et de branches seulement chez les techniciens, mais 20 ans pour le président (naqîb) (art. 11 de la loi 67 de 1974), mais pas de conditions pour les autres membres du conseil, 5 ans d'ancienneté pour les professions du spectacle (10 ans pour le naqîb), aucune ancienneté pour les enseignants, les artistes, les diplômés de commerce, les journalistes (mais dans ce cas, il peut y avoir une exigence d'ancienneté pour les naqîb); parfois encore, les conseils doivent être composés pour moitié d'« anciens », pour moitié de «jeunes » (chez les scientifiques et les infirmières, le seuil étant fixé à plus ou moins 15 ans), etc. Cette question de l'ancienneté exigée pour l'éligibilité aux postes de responsabilité n'est pas anodine; elle révèle la tension entre une conception hiérarchique plutôt associée à la corporation de métier d'ancien régime, et une conception égalitariste républicaine qui est celle du syndicalisme moderne ; la prévalence d'un modèle ou de l'autre dans tel ou tel syndicat, ou la solution de compromis d'une répartition par moitié, me semble pouvoir être considérée comme un indice de la nature et de la finalité du syndicat concerné, ainsi que de la représentation que se font les intéressés de la profession, et leurs dirigeants des intérêts qu'ils défendent.

81 Les modalités d'élection des conseils et des directions syndicales sont au cœur des batailles récentes. En général, les conseils sont élus par l'assemblée générale des adhérents, au niveau des sections locales comme au niveau national. Jusqu'à la réforme introduite par la loi $\mathrm{n}^{\circ} 100$ de 1993, les exigences de quorum étaient très réduites : l'article 18 de la loi 66 de 1974 sur le syndicat des ingénieurs impose d'abord un quorum de $25 \%$ des adhérents à jour de cotisation puis, si ce quorum n'est pas atteint, lors d'une deuxième réunion, la participation de 300 adhérents minimum; pour les assemblées générales de section, cette deuxième limite descend à 50 membres. Lorsque les adhérents n'étaient que quelques milliers, cela ne posait pas de problème : depuis 
quelques années, avec le renversement de majorité dans un certain nombre de syndicats, certains se sont mis à contester le caractère démocratique de directions élues par un nombre trop restreint d'électeurs. La fameuse loi $n^{\circ} 100$ de 1993, si controversée, a donc relevé l'exigence de quorum de manière draconienne, au point qu'il est quasiment impossible à tenir (voir plus loin, ainsi que le texte de la loi en annexe) ${ }^{34}$.

Budget et financement

Les sources de financement sont définies de manière assez précises par la loi, qui consacre un chapitre aux « finances » (al-nizâm al-mâli ou bien mâliyya al-niqâba).

Pour le syndicat des ingénieurs, l'article 45 de la loi $n^{\circ} 66$ de 1974 énumère les ressources suivantes: droits d'inscription et cotisations des membres; droits d'inscription et cotisations des bureaux et sociétés d'ingénierie et de conseil; subventions de l'État; dons et présents; taxe sur les sacs de ciment; taxe sur la production d'acier (hadîd al-taslîh) ; vente des publications du syndicat et rémunération de ses activités ; revenus des timbres fiscaux sur les documents, registres et contrats d'ingénierie ; taxes sur les devis ; revenus des consultations faites par le syndicat ; toute autre ressource légitime (mashrû'a).

Les ressources du syndicat des enseignants sont de nature différente du fait de l'absence de lien de leur activité avec la production de biens : l'article 58 de la loi $\mathrm{n}^{\circ} 79$ de 1969 énumère les ressources suivantes : droits d'inscription ; cotisations annuelles ; revenus des timbres devant être apposés sur diverses demandes adressées au ministère de l'Éducation (inscription dans les différents cycles scolaires, attestations de diplôme, cartes syndicales, formulaires de candidatures); taxes sur diverses primes ou augmentations touchées par les enseignants, sur les droits d'auteur et sur les frais d'impression pour les livres scolaires, sur la construction et l'entretien des bâtiments scolaires, sur l'enseignement privé; une part des amendes imposées en cas de faute professionnelle; revenus des publications du syndicat; aides et dons reçus par le conseil du syndicat ; revenus des placements et investissements.

Si l'on regarde les grandes lignes du budget du syndicat des ingénieurs tel qu'il est publié dans sa revue, en 1993, les diverses ressources fiscales représentent $70 \%$ du total des ressources et les revenus des investissements (qui ressortiraient sans doute de la rubrique «toute autre ressource légitime») $20 \%$, pour un budget de 66 millions de livres égyptiennes (£e), dont... 61 millions sont consacrés à la caisse de retraite ${ }^{35}$. Par comparaison, le syndicat des commerciaux, avec 2,5 fois plus d'adhérents, a en 1994 un budget de 20,5 millions £e, moins de la moitié (9,6 millions) de ses ressources étant d'origine fiscale, et il ne consacre que 16,9 millions £e à la caisse de retraite ${ }^{36}$.

On voit que d'un syndicat à l'autre, l'importance des ressources peut varier très fortement : or, de ces dernières dépendent les services que le syndicat pourra rendre à ses membres. On comprend mieux l'enjeu du contrôle des plus riches d'entre eux, dont un certain nombre sont justement passés sous l'influence des islamistes. Sur le fond, l'importance des ressources que j'ai qualifiées provisoirement de fiscales me paraît mériter réflexion. En effet, tout semble se passer comme si, par le biais des caisses de retraite des syndicats professionnels, certaines catégories de la population bénéficiaient d'un privilège leur permettant de récupérer une part plus importante du produit national; en d'autres termes, comme si l'État opérait une redistribution inégalitaire en faveur des catégories les plus aisées de la population active. Certes, 
d'autres formes de redistribution étaient censées, initialement au moins, privilégier les plus pauvres (soins gratuits, produits subventionnés, etc.).

Sur un autre plan, les scandales et les polémiques se sont multipliés ces dernières années suite à des accusations de détournement de fonds lancées contre les syndicats les plus divers : des agronomes aux guides touristiques en passant par les techniciens ou les professionnels du sport ${ }^{37}$. Plus importantes, plus lourdes de conséquences, les accusations contre le syndicat des médecins (et contre l'action des islamistes dans les syndicats en général) d'utiliser des fonds sous prétexte d'aide à la Bosnie ou d'entraînement des terroristes et, surtout, celles contre les ingénieurs - suite à un rapport de la cour des comptes de 1994 faisant état de fraudes et de détournements des fonds destinés à la caisse de retraite ${ }^{38}-$ qui ont abouti à la mise sous séquestre de leur syndicat en mars 1995.

Il est clair que la dimension financière est un enjeu fort du contrôle des syndicats professionnels, surtout pour ce qui concerne les syndicats les plus riches.

De ce premier survol, il est possible de dégager quelques points d'homogénéité ou d'hétérogénéité de l'ensemble.

D'un côté, un cadre législatif tendant à unifier les réglementations et les fonctionnements : le principe de mobilisation au service de la société et du projet de développement, à côté de cela, la fonction de formation scientifique et professionnelle, un rôle social important en termes de gestion de systèmes de protection et d'aide sociale, l'affirmation de la fonction disciplinaire, sont autant de points communs.

De l'autre, des nuances dans la définition de certaines missions ou dans les conditions d'adhésion et d'exercice, des conditions différentes d'éligibilité aux postes de responsabilité, soulignent une hétérogénéité des héritages et des réalités concrètes des différents groupes concernés face à laquelle l'effort d'unification du législateur ne peut être que vain.

Conflits autour des syndicats professionnels

En 1986, le courant islamique conquiert la majorité des sièges au conseil du syndicat des médecins, en 1987 au conseil des ingénieurs, en 1990 chez les pharmaciens et les scientifiques, en 1992 chez les avocats ${ }^{39}$. Les années quatre-vingt-dix sont jalonnées d'affrontements entre les syndicats les plus prestigieux et le pouvoir, en particulier les journalistes, les avocats, les médecins et les ingénieurs ${ }^{40}$.

93 En 1992, dans l'espoir d'endiguer la montée des islamistes, le pouvoir avait promulgué une première loi (loi $n^{\circ} 98 \mathrm{du}$ 19/07/92) instituant une commission judiciaire pour superviser les élections ${ }^{41}$. Cela n'empêche pas les islamistes de conquérir le syndicat des avocats. Il contre-attaque en promulguant la loi $n^{\circ} 100$ du 18 février 1993 (texte en annexe), qui impose des conditions de quorum draconiennes : $50 \%$ des inscrits doivent participer à l'assemblée générale et au vote à la première convocation, faute de quoi un nouveau rendez-vous est fixé, pour lequel le quorum est abaissé à $33 \%$. Elle prévoit aussi la constitution de bureaux de vote pour chaque regroupement de 500 adhérents. Si ces conditions ne sont pas réalisées, l'administration du syndicat est assurée par une commission présidée par un juge, en attendant l'organisation de nouvelles élections. Des procédures analogues sont appliquées pour les syndicats locaux. De telles exigences ont abouti de fait à bloquer le processus électoral pour de nombreux syndicats ; c'est en particulier la constitution de bureaux de vote sur les lieux de travail qui pose des problèmes à la fois techniques (nécessité de recenser les lieux de travail des membres) 
et politiques (lorsque ces bureaux sont situés dans des casernes ou même des entreprises publiques) ${ }^{42}$.

Cependant, l'établissement des listes électorales ne dépendant que du syndicat échappait encore au contrôle du pouvoir. Une nouvelle loi (la loi $n^{\circ} 5$ du 13 février 1995), durcissant le contrôle administratif sur l'établissement de ces listes et sur l'organisation du scrutin, est votée à la sauvette deux ans plus tard. Ce vote relance le mouvement de protestation. Au printemps 1995, la confrontation se durcit. Après l'arrestation, en décembre, d'un certain nombre de dirigeants des Frères musulmans parmi lesquels de nombreux médecins dont le secrétaire général de leur syndicat, 'Isâm al-Aryân, et la promulgation de la loi $n^{\circ} 5-c^{\prime}$ est la mise sous séquestre, suite à des accusations de malversations financières, du syndicat des ingénieurs, l'occupation de son siège par la police fin février, le vote de défiance de l'assemblée générale du syndicat au naqîb, Hasab Allah al-Kafrawi. Début mai, le siège du syndicat des ingénieurs est occupé par la police. À la fin du mois, une loi sur la presse prévoyant de lourdes condamnations pour les journalistes qui auraient diffusé de fausses informations provoque une réaction massive.

Ces batailles sont essentiellement politiques ; leur enjeu est celui de la démocratie, dans le fonctionnement des syndicats comme dans le pays en général. Deux questions cependant restent taboues : celle du pluralisme syndical et de la liberté d'adhésion. La liberté syndicale est comprise comme le droit de constituer un syndicat pour tout groupe professionnel qui le souhaite, non le droit d'adhérer ou de ne pas adhérer ${ }^{43}$. Les luttes n'ont pas pour objet la situation des professionnels et ne sont guère l'occasion, pour les syndicats, d'exprimer des positions vis-à-vis des réformes économiques en cours. Ce qui ne veut évidemment pas dire que les syndicats ne se mobilisent pas sur les questions économiques : mais il est remarquable qu'ils le font sur un plan pratique, en multipliant les services et les aides, personnels ou professionnels, plus qu'en revendiquant, de la part de l'État ou des entreprises, des mesures d'amélioration de l'emploi ou des salaires ${ }^{44}$, ce qui s'explique très largement par le fait qu'ils ne sont pas structurés sur les lieux de travail.

Conclusion

Dans la première partie de cette étude, je proposais d'orienter ma recherche à partir de deux hypothèses complémentaires.

La première suggérait que l'élargissement du syndicalisme professionnel à des catégories de plus en plus larges de diplômés pouvait être considérée comme l'expression d'une volonté de reconnaissance et de distinction : les luttes autour de la définition des conditions d'adhésion, de l'élargissement de la base des syndicats les plus anciens, l'insistance sur l'usage des titres ou l'invention de nouveaux titres, me paraissent aller dans ce sens. Mais sur un plan plus matériel, les avantages qu'assure l'appartenance à un syndicat professionnel en matière sociale sont aussi une manière d'asseoir une position sociale privilégiée.

Ma seconde hypothèse, complémentaire de la première, considérait les luttes dont ces syndicats sont le théâtre comme mettant en scène anciennes élites professionnelles et nouveaux diplômés, les premières tentant désespérément, dans un combat perdu d'avance, de protéger des positions acquises, menacées par les secondes: les différences de points de vue et de pratiques en matière de hiérarchies professionnelles et d'élection des instances dirigeantes me paraissent révélatrices d'un tel enjeu, à la lumière duquel devrait être reconsidérée l'influence croissante du courant islamiste. 
Pour aller plus loin, deux orientations de recherche devraient être poursuivies, qui auraient en commun de déplacer le regard vers la réalité des groupes socioprofessionnels concernés, plutôt que d'en rester à l'analyse de l'action des syndicats et de leur relation avec le pouvoir, jusqu'à présent dominante :

- la première s'intéresserait à révolution des anciennes professions et aux conséquences de leur différenciation interne croissante : ce sont principalement ces professions qui sont aujourd'hui sous l'emprise du courant islamiste ;

- la seconde s'attacherait à l'étude des syndicats et des groupes moins prestigieux et/ou plus récents, à leur position dans la société et à la relation qu'ils entretiennent avec les autres professionnels.

\section{BIBLIOGRAPHIE}

Abû Ghâzi Nadia Badr al-Dîn, « Le mouvement des syndicats des métiers artistiques », in Démocratie et démocratisation dans le Monde arabe, Dossiers du Cedej, 1992, p. 317-335.

Abû-L-As'ad Muhammad, « Les enseignants égyptiens : un siècle de syndicalisme », Égypte/Monde arabe $\mathrm{n}^{\circ} 18-19,1994$, p. 39-53.

Baer Gabriel, Studies in the Social History of Modem Egypt, University of Chicago Press, 1969.

Bianchi Robert, Unruly Corporatism, Egyptian Associational Life in the 20th Century, New York, Oxford, Oxford University Press, 1989, 267 p.

Botiveau Bernard, «Égypte : crise de l'Ordre des avocats et mobilisation des syndicats professionnels », Maghreb-Machrek n 142, 1993, p. 5-15.

Chiffoleau Sylvia, « Le monopole national d'exercice d'une profession libérale : le cas de la médecine ", Égypte/Monde arabe n 11, 3e trim. 1992, p. 59-76.

Chiffoleau Sylvia, Médecines et médecins en Égypte, Construction d'une identité professionnelle et projet de médicalisation, thèse pour le doctorat en sociologie, Paris, EHESS, 1994.

Dubar Claude, La socialisation, Construction des identités sociales et professionnelles, Paris, Armand Colin, 1991, $278 \mathrm{p}$.

Fergany Nader, « Médecins et ingénieurs sur le marché du travail en Égypte », Maghreb-Machrek n¹46, oct.-déc. 1994, p. 40-47.

Freidson Eliot, Profession of Medicine, Harper and Row, New York, 1970 ; traduction française : La profession médicale, Payot, 1984, 369 p.

Grelon André, Les ingénieurs de la crise. Titre et profession entre les deux guerres, Paris, EHESS, 1986, $461 \mathrm{p}$.

Hanafl Sari, «Ingénieurs et ouverture économique en Syrie et en Égypte : un groupe professionnel en quête d'identité », à paraître in Tiers-Monde, 3e trim. 1995.

Hawâri Anwar, «Les syndicats professionnels », Rapport stratégique arabe 1994, Centre d'études stratégiques d'al-Ahrâm, Le Caire, 1985, p. 385-390. 
Longuenesse Elisabeth, « Ingénieurs au Proche-Orient : Liban, Syrie, Jordanie », Sociétés contemporaines $\mathrm{n}^{\circ} 6$, juin 1991, p. 9-40.

Lucas Yvette, Dubar Claude (éd.), Genèse et dynamique des groupes professionnels, Lille, PUL, 1994. Moore Clément H., « Les syndicats professionnels dans l'Égypte contemporaine : l'encadrement de la nouvelle classe moyenne ", Maghreb-Machrek n 64, 1974, p. 24-34.

Moore Clément H., Images of Development, Egyptian Engineers in Search of Industry, Cambridge, Mass., MIT Press, 1980, 252 p. (1e éd.).

Qandîl Amâni, «Jamâ'ât al-Masâlih (Les groupes d'intérêt) », Rapport stratégique arabe 1986, Centre d'études stratégiques d'al-Ahrâm, Le Caire, 1987 (p. 380-389).

Qandîl Amâni, « Le courant islamique dans les institutions de la société civile : le cas des ordres professionnels en Égypte ", Modernisation et nouvelles formes de mobilisation sociale, Égypte-Turquie, Dossiers du Cedej 1992, p. 173-184.

Qandîl Amâni, «L'évolution du rôle des Islamistes dans les syndicats professionnels égyptiens », Le phénomène de la violence politique, Perspectives comparatistes et paradigme égyptien, Dossiers du Cedej, 1994, p. 281-293.

Rapport stratégique arabe 1987, Le Caire, 1988 (p. 343-357).

Rapport stratégique arabe 1988, Le Caire, 1989 (p. 493-509).

Rapport stratégique arabe 1989, Le Caire, 1990 (p. 456-469).

Rapport stratégique arabe 1991, Le Caire, 1992 (p. 409-423).

Reid Donald, « The Rise of Professions and Professional Organization in Modem Egypt », Comparative Studies in History and Society, XVl/1, 1974, p. 24-57.

Roussillon Alain, « Réforme sociale et politique en Égypte au tournant des années 40 », Égypte/ Monde arabe $\mathrm{n}^{\circ} 18-19,1994$, p. 197-236.

Roussillon Alain (dir.), Entre réforme sociale et mouvement national, Le Caire, Cedej, 1995, 590 p.

Sayyid (al-) Mustafa Kamal, Al-mujtma' wa-l-syâsa fî Misr, dawr jamâ‘ ât al-masâlih fî-l-nizâm al-siyâsî al-misrî (1952-1981), Dâr al-Mustaqbal al-'Arabi, Le Caire, 177 p.

Sayyid (al-) Mustafa Kamal, « A Civil Society in Egypt », MEJ, 47/2, 1993, p. 228-242.

Sayyid (al-) Mustafa Kamal, « Le syndicat des ingénieurs et le courant islamique », MaghrebMachrek n 146, oct.-déc. 1994, p. 27-39.

Springborg Robert, « Professional Syndicates in Egyptian Politics, 1952-1970 », IJMES, IX/3, 1978, p. 275-295.

Tripier Pierre, Du travail à l'emploi. Paradigmes, idéologies et interactions, Bruxelles, éd. de l'Université de Bruxelles, Institut de Sociologie, 1991, 204 p.

Waterbury John, The Egypt of Nasser and Sadat, The Political Economy of two Regimes, Princeton University Press, 1983.

\section{ANNEXES}

Annexe 1 À propos du rôle des syndicats dans le mouvement national égyptien 
Le texte ci-après m'a paru intéressant pour les amalgames qu'il opère entre différents types de mouvements sociaux et pour la représentation du syndicalisme qui en ressort. Il donne sans doute une première idée de la manière dont le courant islamique conçoit le rôle des syndicats dans la société :

- Le syndicalisme doit d'abord être un « mouvement du peuple », dans ses différentes composantes, " contre l'injustice » : c'est ce que révèle la généalogie qui en est proposée, et qui en situe le point de départ dans un mouvement contre un gouverneur « injuste », en 1805, regroupant artisans, ouvriers, commerçants, patrons et ulémas. Le mouvement syndical authentique représente « l'avant-garde du peuple dans sa lutte pour la justice et pour le retour du règne de la loi divine ».

- Aucune distinction n'est faite ni à faire entre syndicalisme professionnel et syndicalisme ouvrier. Les luttes récentes « contre diverses législations dictées de l'étranger » (loi $n^{\circ} 203$ sur le secteur des affaires, remettant en cause le secteur public, le projet de code du travail) ou contre la « loi scélérate » (loi n 100 sur l'organisation des élections syndicales) sont celles du « mouvement syndical égyptien », présenté comme « l'arme la plus efficace du peuple égyptien » contre des gouvernants injustes. Ce faisant, le mouvement syndical se réduit implicitement aux syndicats dirigés par le courant islamique, puisque les autres, y compris la confédération ouvrière, loin de s'opposer au pouvoir, sont globalement contrôlés par lui.

Derrière ces propos, c'est une conception à la fois moralisante, unanimiste et politique du rôle du mouvement syndical qui est proposée, opposant tout le peuple à un État corrompu et représentant des intérêts étrangers, aux antipodes de celle d'un syndicalisme de classe tel qu'il s'est construit, dans ses versions réformistes ou révolutionnaires, dans les pays anciennement industrialisés. En cela, elle n'est pas fondamentalement étrangère à la conception corporatiste dominante à l'époque nassérienne.

\section{E. Longuenesse}

Extrait du journal al-Sha'b du 30/09/94 ('Abd Allah Shâmi)

Dans l'histoire du mouvement national égyptien, le 12 mai 1805 de notre ère (12 Safar 1220 H.) est la véritable date de naissance de l'histoire de l'Égypte moderne et de l'apparition d'un mouvement national moderne. Selon les historiens, 40000 Égyptiens, en ce jour fameux, se sont rassemblés, qui représentaient les patrons, les artisans et gens de métiers et leurs chefs (nuqabâ') ainsi que l'association des ulémas d'al-Azhar. Ils se sont dirigés vers Mahalla al-Kubra, la «Maison du Juge», pour affronter le gouverneur injuste (al-wâli al-zalîm) Khûrshîd Bâsha et proclamer leurs dérisions au Conseil de justice (maglis al-shar'). Selon les normes du monde civilisé de l'époque, ce fut un mouvement politique très avancé qui rassembla tout le peuple : artisans, ouvriers, commerçants, patrons et élite des ulémas d'al-Azhar... Ce mouvement les rassembla non pour qu'ils pillent et sèment le désordre ou la révolte (...), mais pour que le peuple présente ses revendications et qu'une décision soit prise par le juge de la loi divine (qâdi al-shar'). Même selon les normes (miqyâs) d'aujourd'hui, ce mouvement est considéré avec un grand respect, comme le mouvement d'un peuple civilisé (mutahaddir). Voici la conception que le peuple se faisait de la sharî́a et de sa signification (maqâsidu-hâ), telle qu'elle apparaît dans un document publié par les savants d'al-Azhar : « Les peuples, selon les anciens préceptes de la coutume et selon ce qu'imposent les préceptes de la sharî́a islamique, ont le droit de nommer des gouvernants (yuqîmu wulât) et de les 
démettre s'ils s'écartent de la justice et marchent dans la voie de l'injustice, car les souverains injustes se placent hors de la sharîa. La déclaration de 'Umar Makram, chef du mouvement national égyptien de l'époque, soutient ce point de vue quand il déclare : « Les plus dignes de décider (ûlû-l-amr) sont les 'ulamâ' (savants), les spécialistes de la sharî́a (hamalat al-sharî'a) et le souverain juste... et celui-ci (le gouverneur Khûrshîd) est un homme injuste. Or, dans l'ancien temps, il était de coutume que les citoyens (ahl al-balad) écartent les gouverneurs (...), et même le khalife ou le souverain, s'ils tyrannisaient le peuple, étaient écartés et renversés. »

C'est ainsi que le mouvement syndical égyptien a arraché son acte de naissance, comme avant-garde et instrument entre les mains du peuple égyptien pour changer (l'ordre des choses) et imposer le gouvernement fondé sur la loi divine (hukm al-shar') et protéger les droits du citoyen (muwâtin).

Ainsi a avancé le mouvement syndical égyptien : du mouvement des 'ummâl al-'anâbir lors de la révolution de 1919, à la lutte contre les Anglais et au soutien du gouvernement national jusqu'à l'évacuation complète. Ainsi le mouvement syndical a$\mathrm{t}$-il affirmé son rôle quand les syndicalistes ont assumé la charge du développement et de la construction nationale et quand ils ont mobilisé le peuple le jour de la grande traversée, lorsque 9 millions d'ouvriers, de professionnels, d'artisans, de patrons égyptiens ont soutenu une année d'un million de combattants et ont fait le $10 \mathrm{du}$ mois de Ramadan (le 6 octobre 1973, considéré en Égypte comme une grande victoire sur Israël. N.D.L.T.), en ce jour qui fut le début d'une ère nouvelle pour celle région du monde.

Le mouvement syndical comme avant-garde

Aujourd'hui, le mouvement syndical égyptien se dresse, armé des grandes leçons de l'histoire moderne de sa patrie et du puissant élan qu'elle représente, moralement et matériellement, dans la vie du peuple égyptien. C'est là l'explication logique et juste du phénomène qui pèse aujourd'hui sur le mouvement syndical égyptien : la réforme nationale globale fondée sur les principes de la sharîa $a$, le nationalisme égyptien attaché à la solution islamique. Dans le contexte de la crise profonde que traverse notre grande patrie, alors que règnent le désordre politique et la corruption et que le pays s'enfonce dans les impasses de la domination des intérêts américains et sionistes sur le monde, alors que la vie dans toutes ses dimensions est à l'opposé de ce que nous attendions des succès du 10 Ramadan... à travers tout cela, le mouvement syndical revient à son authenticité, comme direction du peuple et moteur du changement au service de la patrie et pour la proclamation du règne de la loi divine (i'lân hukm al-shar').

Le mouvement syndical égyptien s'oppose, depuis environ deux ans, au « mouvement de législation (tashrî) étrangère » qui veut faire des lois (taqnîn) selon les principes du projet étranger visant à régner sur l'Égypte, à limiter sa croissance et à mettre un terme à son rôle dans la région. Les syndicats se sont opposés au mouvement de pillage du secteur public instauré par la loi n 203 de 1991, et à l'attaque concertée dont ils sont l'objet au travers de la loi scélérate (allusion à la loi 100 de 1993, N.D.L.T.) visant à contrôler le mouvement syndical. Aujourd'hui, ils s'opposent à la loi unifiée du travail... et la lutte ne cessera pas avant que le droit (al-haqq) ne l'emporte et que ne soit arraché le droit du peuple, affirmé et proclamé depuis deux cents ans par le mouvement syndical, à écarter les gouvernants, à les renverser et à les remplacer par d'autres, le droit entier à la citoyenneté égyptienne (haqq al-muwâtana sf-misriyya al-kâmil). 
Nous sommes confrontés à une attaque en règle qui a commencé par l'emprise sur le pouvoir en vertu du principe " gouverner les gouvernants de l'Égypte » : ensuite ce pays a été ligoté par les dettes extérieures et intérieures, puis on est allé vers la domination étrangère sur l'économie égyptienne et la transformation de plus de la moitié de la population égyptienne en mendiants... Enfin, une série de lois iniques, dont les principes sont décidés à l'extérieur du pays, mobilisent, pour s'imposer, les forces de la corruption et du désordre politique. Car l'Égypte se trouve assiégée, de l'intérieur et de l'extérieur ; le mouvement de révolte nationale est entravé ; le droit des Égyptiens au développement de leur pays est confisqué, et l'Égypte est privée de l'accès au rang naturel qui est le sien pour une nation en plein essor.

Face à cette attaque en règle, le peuple égyptien fourbit ses armes... La plus efficace est le mouvement syndical égyptien ; les syndicats se sont dressés contre la corruption et ont fait connaître les revendications du peuple en faveur d'une réforme politique globale, ils ont pris en charge la défense du secteur public... et ils ont combattu l'arsenal des lois récentes concoctées pour servir la présence étrangère et son effort de domination sur l'Égypte.

Les principes du mouvement syndical

Le mouvement syndical égyptien, confronté à l'agression du législateur visant à imposer la domination étrangère et à lui donner une apparence de légitimité, se fonde sur les quelques principes de base suivants :

- premièrement, il considère que la situation normale en matière de conception et de promulgation des lois impose - par respect pour les droits nationaux - que les lois émanent des rangs du peuple, là où se trouvent les intéressés, car une loi n'a pas de valeur si elle va à rencontre des intérêts de la majorité du peuple et ne bénéficie pas de son soutien... Les lois injustes et imposées ne vivent pas, elles restent étrangères et défectueuses, jusqu'à ce que le mouvement de reforme les fasse échouer ;

- deuxièmement, la législation égyptienne doit aujourd'hui procéder des principes de la sharîa et de ses objectifs et toute opposition entre la législation et la sharîa mène à l'échec, car la « solution islamique » est aujourd'hui un des fondements d'une position nationale, un acquis du mouvement national depuis qu'il affirme son droit à écarter les gouvernants, à lever les impôts, à refuser l'injustice, à combattre l'étranger, à rejeter l'oppression et à lutter contre la corruption ;

- troisièmement, le mouvement syndical égyptien considère que ce qui lui donne du poids dans sa lutte pour la défense du peuple égyptien et de ses droits naturels, c'est la sincérité de l'allégeance et la clarté de la délégation que donne le peuple à ses représentants, à travers des élections honnêtes qui ouvrent la vote aux fils du peuple bénéficiant de sa confiance, cela pour qu'ils prennent position librement et honnêtement sur les phénomènes de fraude et de corruption.

Sur la base de ce principe, nous avons le droit de refuser la législation pratiquée par des assemblées douteuses, dominées par la corruption et dont la représentativité est mise en doute... Ceux auxquels les masses refusent de confier leurs intérêts, que le peuple prive de sa confiance, ne sont pas habilités à promulguer des lois qui lèsent leurs intérêts. Une réforme politique globale est indispensable pour transformer les cadres de l'action politique en Égypte.

Extrait du journal al-Sha'b, 7/10/94 (2 partie) 
Dans la première partie de cet article, nous avons expliqué pourquoi nous refusions la loi unifiée du travail. Nous l'avons refusée en application des principes posés précédemment. Car les principales idées en ont été élaborées à l'extérieur du pays pour être conformes aux intérêts étrangers. Personne ne peut nier ce fait. Nous l'avons refusée enfin parce qu'elle n'a pas pris la voie logique qui aurait satisfait les forces nationales (wataniyya)... Car le dialogue aurait dû commencer par la base avec les ouvriers, les professionnels, les artisans, les patrons, et ensuite seulement, on serait passé à la législation (taqnîn).

Nous l'avons refusée parce qu'elle ne repose pas franchement sur les concepts de la sharîa qui réglementent les questions du travail, des salaires, des relations de travail entre deux parties : le patron et les travailleurs... La sharî́a dans ce domaine a des visées d'une grande ampleur, qui ont vu le jour puis ont été appliquées bien avant que le monde occidental ne connaisse le principe de l'organisation du travail et des relations professionnelles. La moindre entrave à l'un des principes de la sharî́a serait une raison suffisante pour refuser une loi qui souffrirait d'une telle déficience.

Les devoirs du mouvement syndical

Ainsi vont les choses. Où allons-nous, et que faire ? La réponse est que seul le juste est correct. Même si de telles lois étaient promulguées, le principe éternel en jugerait... "Car l'écume s'évanouit, tandis que demeure ce qui est bon pour l'homme ", et il ne fait aucun doute que ces lois fautives sont une écume éphémère.

Le mouvement syndical égyptien doit prendre l'initiative...Des événements importants l'ont confirmé, qui ne sont que les signes avant-coureurs des changements à venir : les jugements prononcés par la justice égyptienne, qui ont frappé tous ceux qui jouaient avec les droits du peuple, ont condamné la corruption, l'injustice et l'oppression ; les proclamations de l'illustre al-Azhar, qui défend le peuple grâce à la sharî́a et consolide la sharî́a par les forces du peuple; le terrible moment de vérité qui approche à une vitesse effrayante pour déchirer le voile de ce mirage que l'on appelle réforme économique... Les masques sont tombés, tout apparait sous une lumière crue... Ce qui est visé sous ce mot d'ordre de réforme, c'est le pillage du secteur public et le retour des courtiers juifs au pouvoir sur les marchés égyptiens, l'ensevelissement de la livre égyptienne dans le bourbier de la Banque [mondiale] et du Fonds [monétaire international] ! Enfin s'est dévoilé leur grand projet de meurtre démographique : on veut nous faire cesser de croitre et de profiter de notre descendance pour provoquer le grand changement que nous espérons dans nos vies... Tout cela est lié au projet global pour cette région, la normalisation ou la guerre.

Ces événements importants sont pour nous la preuve que l'agression étrangère est un tigre en papier et que ses agents, ses représentants et ses porte-parole, parmi nos compatriotes, fornent un système fragile qui ne peut durer. L'histoire frappe à la porte de l'Égypte. Dans certains moments décisifs, les témoins des événements ont pu croire que le mouvement de l'histoire s'était arrêté... mais 'Ayn Jalût, Marag Dabîq, Shubrakhît, la mobilisation syndicale du 12 Safar 1220 et la grande traversée du 10 Ramadan, tout cela témoigne du fait que le mouvement de l'histoire est un fleuve puissant qui emporte tous ceux qui s'y opposent.

Le mouvement syndical doit prendre l'initiative

Le mouvement syndical doit se rappeler ces leçons et affirmer en permanence sa véritable identité comme avant-garde, combustible et force motrice du mouvement 
national. La Fédération (des syndicats ouvriers), avec l'élite d'al-Azhar et des universités, s'appuyant sur le ferme sodé de la justice égyptienne qui représente la dernière ligne de défense de l'existence et des droits du peuple égyptien, sera le lieu de la prochaine bataille historique pour le changement en Égypte.

Nous avons appelé les syndicats à prendre l'initiative et à constituer, avec l'élite d'alAzhar et des universités, des groupes nationalistes (gamấât wataniyya) chez les patrons et les grands noms de la justice égyptienne, un regroupement national qui propose des formulations nationales légitimes pour toutes ces lois... C'est la revendication urgente du peuple : que nous prenions l'initiative et que nous exercions notre droit naturel à élaborer les lois dont l'action nationale a besoin. De cette formulation nationale, armée de la science et de l'expérience mondiale qui ne s'oppose pas à la justice divine (shar') et à l'intérêt national, les forces nationales passeront sans aucun doute à la prochaine bataille : imposer le changement, susciter les mécanismes de ce changement en termes de lois et de réglementations dont nous réussirions à faire un pacte national légitime $($ shar'). Nous n'avons pas de temps à perdre. Le peuple n'a d'autre espoir, après Dieu (qu'il soit loué), que dans les forces nationales conscientes et dans son avant-garde, les syndicats égyptiens nationaux.

Annexe II : Loi nº100 de 1993

Visant à garantir la démocratie des organisations syndicales professionnelles Au nom du Peuple.

Le Président de la République

L'Assemblée du Peuple a voté la loi dont le texte suit, et nous l'avons promulguée ;

Art. 1 - Les dispositions de cette loi s'appliquent aux syndicats professionnels.

Art. 2 - Pour que l'élection du président (naqîb) et des membres du conseil du syndicat général ou local soit valide, doivent participer au vote la moitié au moins des membres de l'assemblée générale dont les noms sont inscrits sur les registres du syndicat et qui bénéficient du droit de vote, selon les dispositions de la loi de chaque syndicat.

Si ce quorum n'est pas réalisé au terme du processus électoral, les membres de l'assemblée générale sont convoqués une deuxième fois dans les 15 jours, et l'élection sera valide si un tiers de ceux qui ont le droit de voter s'expriment.

Si ce quorum n'est pas réalisé, le naqîb et le conseil assurent leurs fonctions pendant trois mois, au terme desquels il est procédé à de nouvelles élections selon la même procédure, l'élection étant valide aux mêmes conditions.

Art. 3 - Si l'élection du président et des membres du conseil ne se fait pas selon les dispositions définies dans l'article précédent, les fonctions du conseil du syndicat général sont assurées par une commission provisoire, placée sous la présidence du plus ancien président du tribunal d'exception du Caire, et composée des quatre présidents ou vice-présidents les plus anciens de ce tribunal, auxquels sont adjoints les quatre membres les plus anciens ayant droit de vote, selon leur ancienneté dans le syndicat et à condition qu'ils ne soient pas candidats au conseil.

Pour les syndicats locaux, la commission provisoire est composée, sous la présidence du président le plus ancien du tribunal de première instance de la circonscription où se trouve le siège du syndicat, des quatre présidents ou juges les plus anciens du tribunal, auxquels sont adjoints les quatre membres les plus anciens du syndicat local ayant 
droit de vote, selon leur ancienneté dans le syndicat et à condition qu'ils ne soient pas candidats au conseil.

Dans le cas où l'une des personnes mentionnées ci-dessus assurerait la présidence, ou serait membre d'une autre commission provisoire, ou refuserait de siéger, ou ferait l'objet d'une opposition, le second dans l'ancienneté prendrait sa place, puis le suivant.

Cette commission a toutes les fonctions du conseil, et son président celles du naqîb; dans les six mois, elle prend les mesures d'organisation des élections selon les dispositions de cette loi, et la convocation est répétée selon la même procédure jusqu'à ce que le quorum exigé soit atteint.

Art. 4 - Si le poste de naqîb est vacant avant terme dans un syndicat où il est élu par l'assemblée générale, il est remplacé par le plus ancien vice-président selon les circonstances, et l'assemblée générale est convoquée pour élire le naqîb dans un délai de trois mois.

Si le quorum défini dans l'art. 2 n'est pas atteint, une nouvelle assemblée générale est convoquée dans les trois mois selon les dispositions de cette loi. et la convocation est répétée jusqu'à ce que le quorum exigé soit atteint.

Art. 5 - La réunion de l'assemblée générale en vue de l'élection doit se tenir un jour ouvrable, à l'exclusion du vendredi ou des fêtes officielles, et la date doit être annoncée dans deux quotidiens de grande diffusion paraissant en langue arabe.

Art.6 - Les élections se tiennent à tous les niveaux du syndicat au scrutin direct et secret et le vote est supervisé à tous les niveaux par une commission judiciaire, placée sous la présidence du président du tribunal de première instance de la circonscription, composée des quatre membres les plus anciens du tribunal. Si l'un d'entre eux refusait de siéger ou faisait l'objet d'une opposition, le second dans l'ancienneté prendrait sa place, puis le suivant. Cette commission définit le lieu du scrutin, constitue, sous la présidence d'un membre des instances judiciaires, des commissions électorales composées d'au moins trois membres, le président étant choisi sur décision du ministère de la Justice après accord de l'instance judiciaire compétente, de même que la commission tranche pour toutes les questions relatives au processus électoral, sa mission prenant fin avec la proclamation des résultats et le président du tribunal de première instance du sud du Caire proclamant les résultats et le nombre de voix obtenu par chaque candidat. Une commission électorale locale est constituée pour chaque groupe de 500 adhérents au maximum ayant le droit de voter, en contrôlant le lieu de résidence ou le lieu de travail de chaque membre, dans la mesure du possible.

Les commissions électorales sont définies avant la convocation de l'assemblée générale et leur localisation est affichée au siège du syndicat général et des syndicats locaux, avec l'aide des instances administratives concernées.

Art. 7 - Le vote est considéré comme un devoir professionnel auquel on ne doit pas se soustraire. Quiconque s'y soustrait sans excuse valable doit payer, dans l'année suivant l'élection, une cotisation double qui s'ajoute aux ressources du syndicat.

Tout membre du syndicat professionnel doit participer à l'élection du président (naqîb) et des membres du conseil du syndicat. Il surfit qu'il se soit acquitté de ses cotisations avant l'échéance électorale. 
Est inscrit sur le registre des membres actifs tout adhérent (déjà) inscrit sur le registre des non-actifs sur simple demande écrite au naqî̉, par une lettre recommandée avec accusé de réception dès que sont assurées les conditions exigées par la loi pour l'inscription sur le registre des actifs, et il peut voter dès qu'il a versé sa cotisation dans les délais indiqués au troisième alinéa de cet article.

Art. 8 - II est interdit aux conseils des syndicats généraux et locaux et à toutes les organisations syndicales professionnelles de recueillir des fonds ou d'accepter des dons dans un but autre que ceux pour lesquels est constitué le syndicat, ou d'exercer une activité en infraction avec ses objectifs, et il leur est interdit d'utiliser leurs ressources pour d'autres buts que ceux qui fondent son existence.

Tout membre doit demander au tribunal administratif de mettre un terme à toute activité ou mesure en infraction avec les dispositions de cet article, et en cette matière sont appliquées les règles définies dans la loi sur le Conseil d'État.

Art. 9 - Sont annulées toutes les dispositions ayant trait aux élections complémentaires dans l'ensemble des lois en vigueur concernant les syndicats professionnels.

Exceptionnellement, sont tenues les élections complémentaires pour les conseils actuellement en fonction à la date d'application de cette loi, en accord avec ses dépositions.

Si le quorum défini à l'art. 2 de cette loi n'est pas réalisé, les membres restants assurent les fonctions du conseil et l'assemblée générale est convoquée dans les six mois pour élire les remplaçants de ceux dont le mandat a expiré ; la convocation est répétée jusqu'à ce que le quorum sort atteint.

Le mandat de ceux qui sont élus dans ces élections complémentaires prend fin en même temps que celui des autres membres à tous les niveaux du syndicat.

Art. 10 - Toute disposition en contradiction avec cette loi est annulée.

Art. 11 - Cette loi est publiée au Journal officiel et prend effet au lendemain de sa publication.

Cette loi est validée par l'application du sceau de l'État et appliquée comme une de ses lois.

Fait au Caire le 17 février 1993

Hosni Moubarak

Annexe III : Loi n 5 de 1995

Amendant certaines dispositions de la loi n 100 de 1993,

Visant à garantir la démocratie des organisations syndicales professionnelles

Au nom du Peuple,

Le Président de la République

L'Assemblée du Peuple a voté la loi dont le texte suit, et nous l'avons promulguée :

Art. 1 - L'article 6 de la loi nº 100 de 1993, visant à garantir la démocratie des organisations syndicales professionnelles, est remplacé par le texte suivant : 
Art. 6 - Les élections à tous les niveaux syndicaux se tiennent au scrutin direct à bulletin secret.

Toutes les opérations concernant les élections à tous les niveaux du début à la fin sont supervisées par une commission judiciaire, sous la présidence du président du tribunal de première instance de la circonscription où se tient l'élection, composée des quatre plus anciens présidents du même tribunal. Au cas où l'un d'eux refuserait de siéger ou ferait l'objet d'une opposition, le second dans l'ancienneté prendrait sa place, puis le suivant.

Les bureaux de vote sont constitués, sous la présidence d'un des membres des instances judiciaires choisi par décision du ministre de la Justice et après accord de l'instance judiciaire compétente, de trois membres au moins du syndicat, non candidats, choisis par décision du président du tribunal de première instance mentionné dans l'alinéa précédent, et une commission électorale locale est constituée pour tout groupe de 500 membres au maximum ayant le droit de vote, après vérification du lieu de résidence ou du lieu de travail de chaque membre, dans la mesure du possible.

Les commissions locales sont constituées avant la convocation de l'assemblée générale et leur localisation est publiée au siège des syndicats généraux et locaux, avec l'aide des instances administratives concernées.

Art. 2 - Un nouvel article est ajouté à la loi $n^{\circ} 100$ de 1993, sous le numéro 6 bis, dont le texte est le suivant:

Art 6 bis - La commission judiciaire mentionnée à l'article de cette loi en vue du contrôle des élections a les prérogatives suivantes :

1. Elle définit le calendrier de déclaration des candidatures, la date du scrutin, la désignation des commissions électorales, en accord avec la loi.

2. Elle renvoie les registres d'inscription des membres au syndicat général, dans les branches et les sections régionales, pour s'assurer de leur correction, elle examine les listes électorales préparées par le syndicat; le naqîb s'appuie sur elle pour vérifier leur exactitude et leur correspondance avec les registres et avec la réalité ; pour cela, il peut recourir à l'aide de toute personne qu'il jugera compétente.

3. Elle juge des cas d'opposition à l'inscription des noms sur listes électorales ou des non-inscriptions non justifiées, ou des rectifications aux informations mentionnées sur les listes, dans un délai de 7 jours après la publication des listes, et elle transmet ses décisions aux intéressés dans les trois jours qui suivent leur publication.

4. Elle juge de toutes les demandes et plaintes qui lui sont présentées dès l'annonce du début du scrutin et jusqu'à la fin des opérations électorales pour en garantir la conformité à la loi.

5. Les résultais et le nombre de voix obtenu par tous les candidats sont annoncés par le président du tribunal du sud du Caire. La commission électorale prend en main le contrôle des opérations électorales et juge de toutes les questions qui leur sont liées, de la validité ou de l'invalidité des opinions exprimées par les électeurs et de l'application des décisions de la commission judiciaire concernant les demandes et les plaintes qui lui ont été présentées, et sa mission se termine avec la fin des opérations électorales.

Art. 3 - Toute disposition en contradiction avec cette loi est annulée. 
Art. 4 - Cette loi est publiée au Journal Officiel et prend effet au lendemain de sa publication.

Cette loi est validée par l'application du sceau de l'État et est appliquée comme une de ses lois.

\section{Le Caire, le 13 lévrier 1995}

Hosni Moubarak

Annexes traduites de l'arabe par Elisabeth Longuenesse et Samia Rizq

Tableau : Liste des syndicats dits « professionnels »

\begin{tabular}{|c|c|c|c|c|}
\hline Syndicat & $\begin{array}{l}\text { Date } \\
\text { création }\end{array}$ & Fédération & $\begin{array}{l}\mathrm{N}^{\circ} \text { et date de la loi les } \\
\text { régissant }\end{array}$ & $\begin{array}{l}\text { Nb d'inscrits en } \\
1991^{* * *}\end{array}$ \\
\hline Avocats & 1912 & & $n^{\circ} 17,1963$ & 92530 \\
\hline Médecins & 1940 & Prot. santé & $n^{\circ} 45,1969$ & 100520 \\
\hline Dentistes & 1940 & Prof. santé & $n^{\circ} 46,1969$ & 12870 \\
\hline Vétérinaires & 1940 & & $n^{\circ} 48,1969$ & 18260 \\
\hline Pharmaciens & 1940 & Prof. santé & $n^{\circ} 47,1969$ & 29640 \\
\hline Journalistes & 1941 & & $n^{\circ} 76,1970$ & 3370 \\
\hline Ingénieurs & 1946 & $\begin{array}{l}\text { Ing. et prof. } \\
\text { techn. }\end{array}$ & $N^{\circ} 66,1974$ & 192550 \\
\hline $\begin{array}{l}\text { Agronomes et techn. } \\
\text { agricoles* }\end{array}$ & 1949 & & $n^{\circ} 31.1966$ & 225630 \\
\hline Enseignants & 1951 & & $n^{\circ} 319.1951$ & 583100 \\
\hline Comptables** & 1955 & & & \\
\hline Scientifiques & 1955 & & $n^{\circ} 80,1969$ & 34910 \\
\hline Comédiens & 1955 & Prof. artist. & $n^{\circ} 35,1978$ & 1844 \\
\hline Cinéma & 1955 & Prof. artist. & $n^{\circ} 35,1978$ & 3345 \\
\hline Musiciens & 1955 & Prof. artist. & $n^{\circ} 35,1978$ & 4358 \\
\hline $\begin{array}{l}\text { Diplômés des fac. de } \\
\text { commerce }\end{array}$ & 1972 & & $n^{\circ} 40,1972$ & 281330 \\
\hline Professions fœtales & 1973 & & $n^{\circ} 45,1973$ & 25060 \\
\hline
\end{tabular}




\begin{tabular}{|l|l|l|}
\hline $\begin{array}{l}\text { Prof. techniques } \\
\text { appliquées }\end{array}$ & 1974 & $\begin{array}{l}\text { Ing. et prof. } \\
\text { techn. }\end{array}$ \\
\hline
\end{tabular}

\section{NOTES}

1.Son article s'étend longuement sur les péripéties de la bataille, mais il est remarquable qu'elle ne dise rien des problèmes concrets des artistes et du contenu de leurs revendications, autres que celles concernant les modalités d'élection de leur direction et la liberté d'expression.

2.Cf Lucas Y., « Qu'est-ce qu'une sociologie des groupes professionnels ?» in Dubar (éd.), 1994.

3.Voir Roussillon (1995), ainsi que les travaux de B. Botiveau à propos des avocats et des professions juridiques, $\mathrm{S}$. Chiffoleau à propos des médecins, $\mathrm{M}$. Volait à propos des architectes, Gh. Alleaume à propos des ingénieurs, etc.

4.L'ordre est ainsi défini par un juriste français : « De création législative, les ordres professionnels ont pour mission (de service public) le contrôle de l'exercice des professions, discipline incluse ; à cet effet, ils disposent notamment d'un véritable pouvoir juridictionnel. Leur compétence de représentation et de défense des intérêts de la profession les rapproche des syndicats, mais de nombreuses particularités juridiques les en distinguent (obligation d'affiliation, ressort national, pouvoir juridictionnel, impossibilité de conclure des conventions collectives de travail...) »Cf. ENCLOS Ph., "Droit et profession, le cas français », in Lucas, Dubar, 1994, p. 325-328.

5.L'ordre des avocats, dans l'ancien régime européen, était à la fois corporation et confrérie, il était établi à côté d'un parlement. Les ordres d'avocats sont supprimés par la révolution française, en même temps que les corporations et les parlements. Mais le système est rétabli en 1810 pour contrôler les débordements (Encyclopaedia Universalis, article « Avocat »),

6.Voir Freidson E. (1970) à propos des médecins et en particulier, dans son chapitre 2, l'analyse du rôle de la très puissante American Medical Association.

7.Cf. Tripier, p. 143, qui relève que dans ce pays « les ingénieurs se sont vu refuser le statut de profession, le juge ne pouvant pas établir avec certitude qu'ils observeraient une loyauté plus forte à l'égard de leur association professionnelle qu'à celui de la direction de leurs firmes".

8.C'est la période de Vichy qui est à l'origine de la plupart des ordres : médecins, chirurgiens-dentistes, sages-femmes, pharmaciens, experts-comptables, architectes et géomètres-experts ; cependant le projet de création d'un ordre des ingénieurs ne verra jamais le jour. Voir Grelon (dir.), 1986, et en particulier, dans ce même volume, Delamarre D., «Les débuts de la Fédération des associations et sociétés françaises d'ingénieurs diplômés ». p. 159-169.

9.Dans l'Espagne franquiste, les ouvriers doivent obligatoirement adhérer au syndicat, dont les dirigeants sont nécessairement membres du parti ; employeurs et salariés sont membres du même syndicat (Preston ed., Spain in Crisis. The Evolution and Decline of the Franco Regime, Barnes and Noble, New York, 1976, p. 87).

10.New Shorter Oxford English Dictionary, article « Syndicate».

11.Exemples dans la presse : Middle East Times, 19/02/95 : « Parliament railroads through union law », après quoi l'article commence ainsi : «A controversial amendment to the law 
governing elections in Egypt's trade syndicates was rushed through parliament on $12 \mathrm{Feb}$ despite angry opposition from the syndicates themselves... » Plus loin, il est fait mention des «syndicate boards » mais des « union activists », etc. AI-Ahrâm Hebdo, 08/03/95 : Sous le titre. «Syndicat des ingénieurs, la bataille autour d'un bilan », on lit : « La mise sous contrôle judiciaire de l'Ordre des ingénieurs par un tribunal cairote... (les islamistes) majoritaires au sein de la direction dénoncent un complot (...) dans le but de s'emparer du syndicat... Après le syndicat des avocats (...), c'est au tour de l'Ordre des ingénieurs de connaître la division... » Dans un article d'Amani Qandil publié dans un Dossier du Cedej en 1992 (voir Bibliographie), niqâba est systématiquement traduit par ordre et le syndicat des enseignants (niqâba al-mu'allimîn) devient ordre des instituteurs; dans un autre texte publié en 1994 (id.), il est traduit par syndicat. De même, dans l'article d'Abul-As'ad (1994), les deux termes sont utilisés alternativement (p. 47).

12.Voir Springborg (1978), p. 284, qui cite Adib Dimitri et Adil Sami Nasr dans al-Kâtib $\mathrm{n}^{\circ} 6$ (septembre 1966).

13.Le terme étant transcrit tel quel.

14.La justice est, plus que tout autre secteur d'activité, influencée par le modèle français. Plus largement, les élites professionnelles et intellectuelles sont largement francophones et c'est sans doute ce qui explique que le modèle de l'ordre professionnel ait ainsi prévalu.

15.On peut ajouter le syndicat des artistes peintres créé la même année, en 1976, mais celui-ci rentre dans la catégorie des métiers artistiques, à côté des comédiens et musiciens.

16.Voir par exemple, pour le cas des médecins, Chiffoleau, 1994.

17.Source : entretiens au Club des commerciaux.

18.Ce qui, étant donné les circonstances, ne signifie pas qu'il le fasse, mais ceci est un autre problème, lié à la nature du système socio-politique plus qu'à cette de l'organisation syndicale.

19.II est possible de faire partie de plusieurs syndicats du spectacle. Il l'a été - mais cela ne semble plus être le cas - d'appartenir à la fois au syndicat des enseignants et à celui des ingénieurs ou même, exceptionnellement, au syndicat des techniciens et à celui des ingénieurs, etc.

20.Ainsi de la grande grève de Kafr al-Dawwar en octobre 1994 (cf. Clément F, " Péripéties et vicissitudes de la libéralisation du marché du travail en Égypte », Égypte/ Monde arabe $\mathrm{n}^{\circ}$ 20, 4e trim. 1994, p. 143-153.

21.Une exception, les banques, où ils peuvent représenter $80 \%$ des membres du conseil.

22. Voir la revue al-'Amal n² 285, juin 1995, p. 24 et s.

23.La loi date de 1969 et n'a pas été amendée depuis, malgré la suppression de l'Union socialiste arabe.

24.Même si c'est sans doute assez formel, cela me paraît symboliquement important.

25.Pour les médecins, la protection du titre n'est pas mentionnée dans la législation syndicale mais dans celle - ancienne puisque datant de 1954 - réglementant l'exercice de la médecine ; de même, les registres sont sous double contrôle du ministère de la Santé et du syndicat.

26.Voir, dans un autre contexte, les batailles autour de la protection du titre d'ingénieur en France et en Europe dans Grelon A., ingénieurs de la crise, Paris, MSH, 1987. En France, « la profession d'ingénieur n'existe pas comme telle » et si « la Commission du titre d'ingénieur contrôle la délivrance des diplômes et l'usage du titre 
qu'il confère, spécifié par l'école », elle «n'empêche personne de se désigner et d'être embauché comme ingénieur » (Gadea, Bercot, « La formation continue et l'accès au titre d'ingénieur en France ». in Lucas, Dubar, éd., Genèse et dynamique des groupes professionnels, PUL, Lille, 1994).

27.Voir les propositions de Johnson T. dans « The Internationalisation of Expertise » in Lucas, Dubar (ed), 1994 (p. 187-201), et dans « The State and the Professions » in Giddens and Mackenzie (ed), Social Class and the Division of Labour, Cambridge University Press, 1982.

28.Cf. l'intervention de l'architecte Milâd Hanna à la Conférence des syndicats professionnels du 14 avril 1995.

29.Les choses sont un peu plus compliquées dans le cas des avocats - pour lesquels, à côté du registre général, existent des registres spécifiques auprès des différents types de tribunaux (art. 10, loi 17 de 1983) - et dans celui des médecins...

30.Art. 10 du règlement intérieur, décret $n^{\circ} 94$ de 1974 du ministère des Finances. 31.Voir al-Tugârî, journal du syndicat du Caire, février 1994 (un seul numéro est paru jusqu'à présent).

32.À titre d'exemple, le chapitre 7 de la loi $n^{\circ} 66$ de 1974 sur le syndicat des ingénieurs, la section 5 de la loi ${ }^{\circ} 79$ de 1969 sur le syndicat des enseignants, sont consacrés à la " caisse de retraite".

33.Le syndicat des professions techniques a une section par province, plus trois sections dans les plus grosses concentrations ouvrières, à Mahalla al-Kubra, Kafr alDawwâr et Nagaa Hammâdi. Le syndicat des guides touristiques a des sections dans les provinces à forte activité touristique seulement.

34.On peut signaler par ailleurs que certains syndicats se sont regroupés en fédérations professionnelles : la fédération des professions de santé regroupe les médecins, dentistes..., la fédération des professions techniques regroupe les professions techniques appliquées et les ingénieurs, la fédération des professions artistiques regroupe comédiens, musiciens, travailleurs du cinéma. La question de la création d'une fédération des syndicats professionnels est posée depuis les années soixante-dix ; le fait qu'elle n'ait jamais vu le jour est sans doute dû au problème du mode de représentation des syndicats, donc des tendances politiques, et à la bataille que se livrent pouvoir et opposition pour leur contrôle.

35.Mahalla al-Muhandisîn $\mathrm{n}^{\circ}$ 444, février 1993.

36.Document daté du 31/12/94, communiqué par le syndicat.

37.Fin 1994, le syndicat des agronomes (al-Sha'b, 20/12/94) est accusé de dépenser l'argent en indemnités mirobolantes sous prétexte de congrès ; au même moment, la caisse de retraite fait faillite. De même au syndicat des professionnels du sport, en août 94 (al-Wafd, 06/08/94), ou au syndicat des guides touristiques : procès qui se termine cependant par un non-lieu à la même époque ( 'Arabi 08/08/94).

38.Cf. al-Ahrâm Hebdo du 08/03/95, al-Ahrâm Weekly du 02/03/95.

39.Voir Amâni Qandil, 1992, p. 174.

40.Voir les comptes rendus de ces événements dans la chronique annuelle du Rapport Stratégique publié par le Centre d'études stratégiques d'al-Ahrâm.

41.Ibid., p. 333.

42.Voir le compte rendu relativement détaillé des péripéties concernant ces élections, en particulier pour les médecins, les ingénieurs et les commerciaux, donné par Anwar al-Hawâri dans le Rapport stratégique de 1994.

43.Hawari, 1995, p. 390. 
44. Voir Longuenesse, Colloque franco-égyptien de science politique, Paris, mai 1995. Les exceptions existent mais sont peu nombreuses : revendication d'une amélioration des primes chez les scientifiques, de garanties de sécurité pour les guides touristiques, etc.

INDEX

Mots-clés : syndicalisme, travail, corporatisme, société

\section{AUTEUR}

\section{ÉLISABETH LONGUENESSE}

Cedej-CNRS 